\title{
Modelling of compaction in planetesimals ${ }^{\star}$
}

\author{
Wladimir Neumann ${ }^{1}$, Doris Breuer ${ }^{1}$, and Tilman Spohn ${ }^{1,2}$ \\ ${ }^{1}$ Deutsches Zentrum für Luft- und Raumfahrt (DLR), Institut für Planetenforschung, Planetenphysik, Rutherfordstr. 2, 12489 Berlin, \\ Germany \\ e-mail: wladimir.neumann@dlr.de \\ 2 Institut für of Planetologie, Westfälische Wilhelms-Universität Münster, Wilhelm-Klemm-Str. 10, 48149 Münster, Germany
}

Received 15 February 2014 / Accepted 13 May 2014

\section{ABSTRACT}

\begin{abstract}
Aims. Compaction of initially porous material prior to melting is an important process that has influenced the interior structure and the thermal evolution of planetesimals in their early history. On the one hand, compaction decreases the porosity resulting in a reduction of the radius and on the other hand, the loss of porosity results in an increase of the thermal conductivity of the material and thus in a more efficient cooling. Porosity loss by hot pressing is the most efficient process of compaction in planetesimals and can be described by creep flow, which depends on temperature and stress. Hot pressing has been repeatedly modelled using a simplified approach, for which the porosity is gradually reduced in some fixed temperature interval between $\approx 650 \mathrm{~K}$ and $700 \mathrm{~K}$. This approach neglects the dependence of compaction on stress and other factors such as matrix grain size and creep activation energy. In the present study, we compare this parametrised method with a self-consistent calculation of porosity loss via a creep related approach.

Methods. We use our thermal evolution model from previous studies to model compaction of an initially porous body and consider four basic packings of spherical dust grains (simple cubic, orthorhombic, rhombohedral, and body-centred cubic). Depending on the grain packing, we calculate the effective stress and the associated porosity change via the thermally activated creep flow. For comparison, compaction is also modelled by simply reducing the initial porosity linearly to zero between $650 \mathrm{~K}$ and $700 \mathrm{~K}$. As we are interested in thermal metamorphism and not melting, we only consider bodies that experience a maximum temperature below the solidus temperature of the metal phase.

Results. For the creep related approach, the temperature interval in which compaction takes place depends strongly on the size of the planetesimal and is not fixed as assumed in the parametrised approach. Depending on the radius, the initial grain size, the activation energy, and the initial porosity and specific packing of the dust grains, the temperature interval lies within $500-1000 \mathrm{~K}$. This finding implies that the parametrised approach strongly overestimates compaction and underestimates the maximum temperature. For the cases considered, the post-compaction porous layer retained at the surface is a factor of 1.5 to 4 thicker for the creep related approach. The difference in the temperature evolution between the two approaches increases with decreasing radius and the maximum temperature can deviate by over $30 \%$ for small bodies.
\end{abstract}

Key words. conduction - planets and satellites: composition - planets and satellites: formation - planets and satellites: interiors minor planets, asteroids: general - meteorites, meteors, meteoroids

\section{Introduction}

Investigations of the structure of chondritic meteorites reveal significant variations in the average porosity between samples (Wilkinson et al. 2003), suggesting that they formed at different depths in the respective parent body. Furthermore, the average porosities of less than $27 \%$ (0-27\% for $\mathrm{H}$ chondrites, $0-14 \%$ for L chondrites, and $0-18 \%$ for LL chondrites, see Wilkinson et al. 2003) are rather small compared to the average porosity of a model structure with a random loose or a random close packing of grains. Random packings should actually be expected for objects that accreted from dust in the protoplanetary nebula and that did not experience any kind of compaction, i.e. values of the porosity range between 36\% and 44\% (Dullien 1992).

The measured average porosities of the meteorites, however, indicate that processes such as compaction and metamorphism have modified the parent body. Compaction, i.e. the decrease of the porosity below the equilibrium value (see below), can be caused by mechanical processes (such as stress, plastic or brittle deformation, and fracture evolution) and/or by geochemical

* Appendix is available in electronic form at http: //www . aanda.org processes (such as dissolution and reprecipitation). Thus, the porosity of the random packings can be considered as initial porosity of the superficial material that accumulates at the surface during the accretion of a planetesimal.

Assuming random packing of particles that accreted at the surface of a planetary object, the porosity of that material depends on its structural parameters. The most important parameters are the grain size, the packing, the particle shape and the grain size distribution. The average or total porosity of an object is defined as the ratio of pore volume to the total bulk volume and can be expressed as a fraction or as a percentage. We note that the fraction value does not contain any information about distribution, sizes, or connectivity of the pores. Thus, objects with equal total porosity can have different physical properties. The porosity of randomly packed material is in equilibrium if it cannot be changed by tapping and shaking and without applying any pressure or increasing temperature. The value at which such an equilibrium state is achieved depends on the frictional and cohesive forces between the grains that are proportional to the exposed surface area. Per unit volume, the exposed surface area is inversely proportional to the grain size. Thus, coarse grain structures stabilise at a lower porosity than finer grain structures assuming the same mass. If the grain size increases above $50 \mu \mathrm{m}$, 
the frictional and cohesive forces decrease and a limit of the porosity is reached at a value of $\phi \approx 0.399$; for larger grain sizes this limit remains constant. This value represents a lower boundary for the porosity of randomly packed spheres (due to shaking and tapping only). Once non-spherical grains are considered, the porosity of the non-frictional limit changes, i.e. random packings of angular grains (e.g. cubes, cylinders, discs) attain equilibrium porosities higher than 0.399 . However, a mixture of two grain sizes, for example, leads to smaller porosities than those of the samples with the involved constant grain sizes (see e.g. Coogan $\&$ Manus 1975). In contrast, the equilibrium porosity of ordered packings is independent of the grain size and depends simply on the choice of the packing. In the present study, we will use ordered packing based on the work by Kakar \& Chaklader (1967) as this allows us to model self-consistently the initial porosity and the compaction process for selected packings.

In general, compaction can be considered as a two-stage process. Owing to the radial growth during the accretion, accreted particles are subsequently covered by fresh material and experience increasing lithostatic pressure. This means that the randomly or ordered packed grains can become closer and compact to the limit of $\phi \approx 0.4$ (see e.g. Guettler et al. 2009). This is a purely self-gravitational effect that does not require any temperature increase above the nebula temperature, and is referred to as cold pressing. Further compaction below the porosity of $\phi \approx 0.4$ is due to the so-called hot pressing or sintering, which can be described by plastic deformation of the grains by thermally activated creep processes. It depends on the temperature-dependent viscosity of the material and the effective stress acting on a dust grain. The effective stress depends on the geometric packing of the particles and the lithostatic pressure. Thus, compaction is in particular governed by temperature and stress. Because the pressure and thus the effective stress in a planetesimal increases with depth, shallow layers require a higher temperature than deep layers to compact by the same degree.

In the literature, the process of porosity loss by sintering in planetesimals is commonly modelled using a parametrised approach, for which compaction occurs at the fixed temperature interval of 670-700 K (e.g. Hevey \& Sanders 2006; Sahijpal et al. 2007; Gupta \& Sahijpal 2010; Solano et al. 2012). The approach dates back to a paper by Yomogida \& Matsui (1984). In that work, the experimental results from Schwenn \& Goetze (1978) were combined with a geometric approach of the calculation of the effective stress on dust grains from Kakar \& Chaklader (1967) and Rao \& Chaklader (1972) to model selfconsistently compaction of planetesimals with radii of $\leq 100 \mathrm{~km}$. The hot pressing experiments by Schwenn \& Goetze (1978) with olivine have been reproduced to model the evolution of $\log (1-\phi)$ (where $\phi$ is the porosity; see also Eq. (9)) using an activation energy of $E=85 \pm 29 \mathrm{kcal} \mathrm{mol}^{-1}\left(E=356 \pm 121 \mathrm{~kJ} \mathrm{~mol}^{-1}\right)$. In their numerical simulations, however, Yomogida \& Matsui (1984) used $E=45 \mathrm{kcal} \mathrm{mol}^{-1}\left(E=188 \mathrm{~kJ} \mathrm{~mol}^{-1}\right)$ for temperatures below $700 \mathrm{~K}$. This value was chosen in order to obtain compaction at low temperatures to reconcile the meteoritic evidence of compaction even with inefficient heating by long-lived isotopes ${ }^{40} \mathrm{~K},{ }^{232} \mathrm{Th},{ }^{235} \mathrm{U}$, and ${ }^{238} \mathrm{U}$. Heating by ${ }^{26} \mathrm{Al}$ and ${ }^{60} \mathrm{Fe}$ was not considered in their study and most bodies considered by Yomogida \& Matsui (1984) had maximum temperatures of $\leq 700 \mathrm{~K}$. Yomogida \& Matsui (1984) concluded that the sintering temperature, i.e. the temperature at which sintering starts to become important, is around $600-650 \mathrm{~K}$. In this manner they artificially favoured compaction at low temperatures. The value of $E=45 \mathrm{kcal} \mathrm{mol}^{-1}\left(E=188 \mathrm{~kJ} \mathrm{~mol}^{-1}\right)$ lies clearly outside of the interval given by Schwenn \& Goetze (1978) for olivine dominated material and is too low for an H-chondritic composition. Typical values of the activation energy for creep processes are $295-530 \mathrm{~kJ} \mathrm{~mol}^{-1}$ in olivine dominated mantle rocks (Karato 2013), 270-720 $\mathrm{kJ} \mathrm{mol}^{-1}$ for enstatite powders (Bystricky et al. 2011; Karato 2013), and 49-130 kJ mol ${ }^{-1}$ for ice (Goldsby \& Kohlstedt 2001; Durham et al. 2001).

Considering the assumptions in Yomogida \& Matsui (1984), their conclusions about the sintering temperature are valid. However, their findings have been simplified and generalised in other studies. Hevey \& Sanders (2006) referred to Yomogida \& Matsui (1984) and incorporated compaction at $700 \mathrm{~K}$ in their simulations. Later, Sahijpal et al. (2007) and Gupta \& Sahijpal (2010) included a gradual reduction of porosity in the temperature range of $670-700 \mathrm{~K}$ in their models and Solano et al. (2012) used the same approach to model compaction of Vesta. Apart from the models by Henke et al. (2012) and Neumann et al. (2012), who consider compaction of chondritic planetesimals as a thermally activated creep process (and possibly the calculations of Akridge et al. 1997; Senshu 2006), most studies use the simplified parametrised approach based on Yomogida \& Matsui (1984) and allow compaction in a rather small temperature window at $\approx 700 \mathrm{~K}$ (e.g. Akridge et al. 1998; Hevey \& Sanders 2006; Sahijpal et al. 2007; Gupta \& Sahijpal 2010) to study the insulating effect of regolith on the thermal evolution. Other studies which do not consider compaction explicitly, but discuss its effects on the results, still refer to some of the publications mentioned above (e.g. Elkins-Tanton et al. 2011; Moskovitz \& Gaidos 2011).

In a previous study (Neumann et al. 2012), we investigated compaction of planetesimals with radii of $\leq 120 \mathrm{~km}$ by hot pressing using a creep-related approach combined with accretion. We compared the thermal evolution of initially consolidated bodies with that of initially porous ones and concluded that the maximum temperature in initially highly porous planetesimals is significantly higher than in consolidated bodies. This effect is particularly pronounced (temperature increase by up to $300 \%$ ) for small planetesimals with radii of less than a few $\mathrm{km}$. We also concluded that compaction starts at temperatures around $600 \mathrm{~K}$ (at high pressures) and could proceed to the temperatures above $1200 \mathrm{~K}$ (at low pressures). These findings are consistent with the results of Henke et al. (2012). Nevertheless, a systematic study is lacking that examines whether compaction achieved by the reduction of the porosity in a fixed temperature interval is an acceptable approximation and that discusses the implications of this approach.

In the present study, we compare two approaches to model compaction of planetesimals via hot pressing. The first one is based on the plastic deformation of dust grains and is referred to as the "creep related approach" in the following. The second one is based on the linear reduction of the porosity from its initial value to zero in the temperature interval $650-700 \mathrm{~K}$, and is referred to as the parametrised approach. We show that the parametrised approach overestimates compaction by hot pressing due to the usage of a specific temperature interval. Furthermore, we investigate in which way ordered packing of grains, grain size, and activation energy influence the compaction process.

\section{Model}

We use the model of thermal evolution and differentiation of planetesimals described in Neumann et al. (2012) and Neumann et al. (2013). The energy balance is solved in a spherically symmetric planetesimal, using a 1D heat conduction equation 
Table 1. Parameter values for the computation of the effective stress.

\begin{tabular}{lrrrrrr}
\hline \hline & & SCP & ORP & RHP & BCCP1 & BCCP2 \\
\hline$Z$ & - & 6 & 8 & 12 & $Z_{1}=8$ & $Z_{1}+Z_{2}=14$ \\
$\alpha_{1}$ & - & $\frac{\pi}{4}$ & $\frac{\pi}{2 \sqrt{3}}$ & $\frac{\pi}{\sqrt{2}}$ & $\frac{2 \sqrt{3}}{7} \pi$ & $\frac{5 \sqrt{3}}{14} \pi$ \\
$\beta_{1}$ & - & 8 & $4 \sqrt{3}$ & $4 \sqrt{2}$ & $\frac{32 \sqrt{3}}{9}$ & $\frac{32 \sqrt{3}}{9}$ \\
$r_{0}$ & - & $\left(\frac{3}{4 \pi}\right)^{\frac{1}{3}}$ & $\left(\frac{3}{4 \pi}\right)^{\frac{1}{3}}$ & $\left(\frac{3}{4 \pi}\right)^{\frac{1}{3}}$ & $\left(\frac{3}{4 \pi}\right)^{\frac{1}{3}}$ & $\left(\frac{3}{4 \pi}\right)^{\frac{1}{3}}$ \\
$r_{\mathrm{c}}$ & - & 0.72 & 0.64 & 0.66 & 0.64 & 0.67 \\
$D_{0}$ & $\%$ & 52.36 & 60.46 & 74.05 & 68.02 & 68.02 \\
$D_{\mathrm{c}}$ & $\%$ & 96.51 & 83.51 & 96.41 & 93.95 & 99.45 \\
$\phi_{0}$ & $\%$ & 47.64 & 39.54 & 25.95 & 31.98 & 31.98 \\
$\phi_{\mathrm{c}}$ & $\%$ & 3.49 & 16.49 & 3.59 & 6.05 & 0.55 \\
\hline
\end{tabular}

Notes. See also Table A.1.

where density, thermal conductivity, heat source density, and radius are functions of the porosity. The body is heated by ${ }^{26} \mathrm{Al}$ and ${ }^{60} \mathrm{Fe}$ and is initially porous (we note that in the current study we adopt a different abundance of ${ }^{60} \mathrm{Fe}$ than in our previous papers; see Table 2). The porosity changes with time because of compaction via hot pressing. The hot pressing is implemented based on the experimental results from Schwenn \& Goetze (1978) and on the geometric deformation theory from Kakar \& Chaklader (1967) and Rao \& Chaklader (1972) (also utilised by Yomogida \& Matsui 1984). In Neumann et al. (2012, 2013) we considered one possible (orthorhombic) packing of equally sized spheres and a constant initial porosity of $\phi_{0}=0.4$ for the computation of the effective stress between dust grains in a compacting system. A similar model, but with an initial porosity of $\phi_{0}=0.5$, was used by Henke et al. (2012). In the present study, we consider four basic systems of packing in order to compare the resulting thermal evolution and porosity distribution with the ones from the parametrised approach, i.e. compaction occurs in the temperature interval 650-700 K. While the thermal evolution including the dependence of density, thermal conductivity, heat source density, and radius on porosity and the setup of the numerical model was described thoroughly in Neumann et al. (2012, 2013), we describe here in more detail the theoretical basis on the modelling of the porosity loss.

\subsection{Theoretical approach to the modelling of compaction}

One of the most common forms of plastic flow is power law creep, which describes the relation between the steady-state strain rate $\mathrm{d} \varepsilon / \mathrm{d} t$ and the effective stress during hot pressing,

$\frac{\mathrm{d} \varepsilon}{\mathrm{d} t}=C \sigma_{1}^{n}$

where $\sigma_{1}$ is the effective stress acting on the material during creep, the stress exponent $n$ is a material constant, the factor $C=$ $C(T)$ is a function of temperature, and $t$ is time. The strain rate during densification of powder can be given in terms of the rate of change of the relative density $D=1-\phi$ (or of the porosity $\phi$ ) as

$\frac{\mathrm{d} \varepsilon}{\mathrm{d} t}=-\frac{\mathrm{d} l}{l \mathrm{~d} t}=\frac{1}{D} \frac{\mathrm{d} D}{\mathrm{~d} t}$

This is an approximation of the strain rate during densification of a powder compact of the height $l$ in a die and can be applied in the first approximation to small planetary bodies. By setting

$$
\frac{1}{D} \frac{\mathrm{d} D}{\mathrm{~d} t}=C \sigma_{1}^{n}
$$

and

$\frac{1}{D} \frac{\mathrm{d} D}{\mathrm{~d} t}=\frac{\partial \log (1-\phi)}{\partial t}$

we obtain

$\frac{\partial \log (1-\phi)}{\partial t}=C \sigma_{1}^{n}$,

where $C$ and $n$ need to be determined experimentally and $\sigma_{1}$ needs to be computed with respect to the geometry of the material on a small scale. Thereby, $\sigma_{1}$ changes with the relative density during compaction. It is widely accepted that the effective stress during hot pressing is not equal to the applied stress $\sigma_{0}$ and depends on the porosity $\phi$. Often, an inversely linear dependence $\sigma_{1}=\sigma_{0} D^{-1}$ on the relative density is used, suggested by Farnsworth \& Coble (1966) and so any specific intrinsic geometry of the material is neglected.

The intrinsic geometry is accounted for in the approach of Rao \& Chaklader (1972),

$\sigma_{1}=\sigma_{0} \alpha_{1}^{-1}\left(D^{2 / 3} \beta_{1}^{2 / 3} r^{2}-1\right)^{-1}$

which we adopt in the present study. Here, $\sigma_{0}$ is the lithostatic pressure inside a planetesimal, the parameters $\alpha_{1}$ and $\beta_{1}$ are constant and reflect the assumptions on the packing of dust grains, and $r$ is the numerical grain radius (varying between $r_{0}$ and $r_{\mathrm{c}}$; see Table 1 and Appendix A for more details). Figure 1 shows the relative stress $\sigma_{1} / \sigma_{0}$ as a function of the relative density $D$ calculated according to Eq. (6). Prior to compaction (having a high porosity and a low relative density), the value of the effective stress $\sigma_{1}$ is several orders of magnitude higher than that of the lithostatic pressure. During the ongoing consolidation of the material, the relative stress approaches the value 1 meaning that the effective stress converges against the lithostatic pressure if the relative density approaches 1 (or the porosity approaches 0 ).

In the study by Kakar \& Chaklader (1967) four basic systems of packing are considered, defined by the geometric arrangement of spherical grains and by the coordination number (i.e. the number $Z$ of the contact points, an average value for equally sized spheres). In the simple cubic packing (SCP) every grain has four neighbours in a horizontal layer and two additional neighbours in the layers above and below. Orthorhombic (ORP) packing corresponds to six neighbours in a layer (plus two above and below). The rhombohedral packing (RHP) has coordination number 12 and the body-centred cubic packing (BCCP) 8. A geometric theory was introduced by Kakar \& Chaklader (1967) in order to treat the deformation of the spheres involved in any of these four 


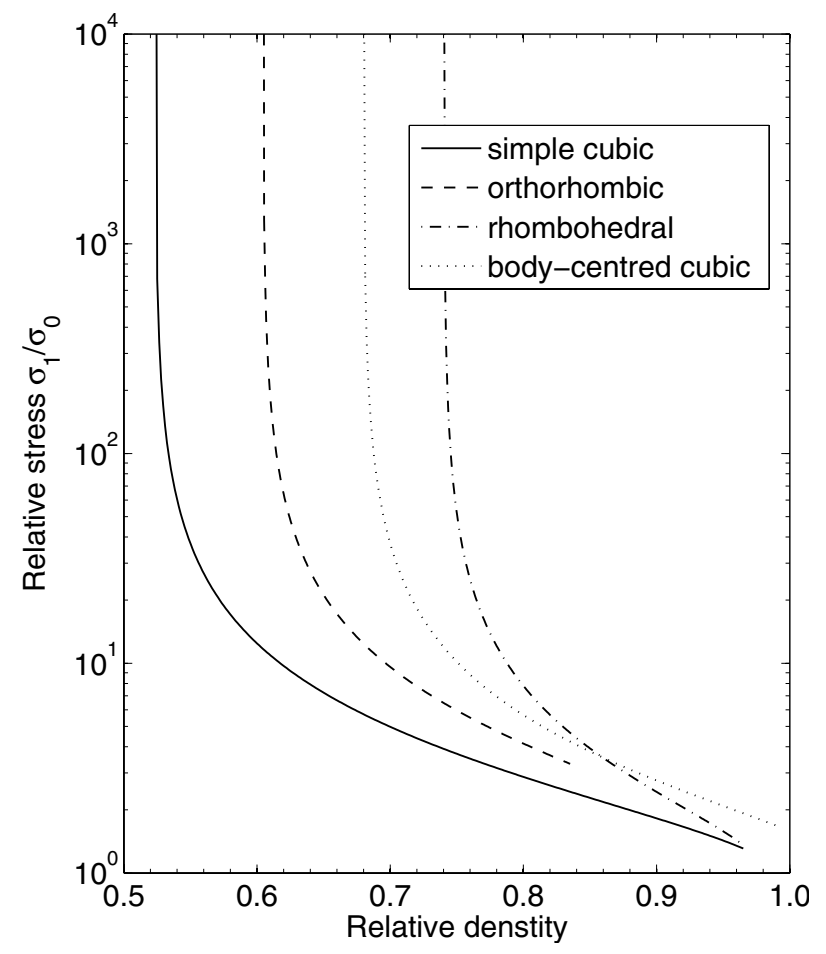

Fig. 1. Relative stress $\sigma_{1} / \sigma_{0}$ for the adopted four basic systems of packing plotted against the relative density $D=1-\phi$ (after Rao \& Chaklader 1972, their Fig. 6). The lines are truncated at the respective critical density $D_{\mathrm{c}}$ (see Table 1), above which the effective stress is computed using a linear relationship between the value at $D_{\mathrm{c}}$ and the lithostatic pressure.

packings and thus the loss of porosity in a dust sample. We note that according to this theory, the deformation that corresponds to $\mathrm{BCCP}$ is a two-stage process. We refer to the two stages as BCCP1 and BCCP2 (see Appendix A for the detailed information). Based on the above model, Rao \& Chaklader (1972) introduced a method for the computation of the effective stress acting on a dust particle (see Eq. (6)). Combination of the effective stress from Eq. (6) with Eq. (5) yields

$\frac{\partial \log (1-\phi)}{\partial t}=C\left(\frac{\sigma_{0}}{\alpha_{1}\left(D^{\frac{2}{3}} \beta_{1}^{\frac{2}{3}} r^{2}-1\right)}\right)^{n}$.

There are two parameters $C=C(T)$ and $n$ which depend on the temperature and on the material properties (such as activation energy). Experimental data for olivine is available from the laboratory experiments of creep of olivine during hot-pressing performed by Schwenn \& Goetze (1978). They provide a fit obtained for spherical olivine grains with radii $\leq 50 \mu \mathrm{m}$ :

$C(T)=B b^{-m} \mathrm{e}^{-\frac{E}{R T}}, n \approx \frac{3}{2}$.

Here, $B$ is a constant factor between $5.06 \times 10^{-19}$ and $1.71 \times$ $10^{-18} \mathrm{~m}^{3} \mathrm{~Pa}^{-3 / 2} \mathrm{~s}^{-1}, m$ is the grain size exponent, $E$ is the activation energy in $\mathrm{kJ} \mathrm{mol}^{-1}, R$ is the gas constant in $\mathrm{kJ} \mathrm{mol}^{-1} \mathrm{~K}^{-1}$, and $b$ is the grain radius in $\mathrm{m}$ (see Table 2 for the adopted values). Consequently, we obtain

$$
\frac{\partial \log (1-\phi(r))}{\partial t}=B b^{-3} \sigma_{1}^{\frac{3}{2}} \mathrm{e}^{-\frac{E}{R T}}
$$

Apart from different numbers of contact points, all four packings have different initial porosities $\phi_{0}$ (or initial densities $D_{0}$ )
Table 2. Physical parameter values used for the models.

\begin{tabular}{llcl}
\hline \hline Quantity & \multicolumn{1}{l}{ Symbol } & Unit & Value \\
\hline Metal mass fraction & \multicolumn{1}{l}{$x_{\mathrm{Fe}}$} & $\%$ & 21.9 \\
Silicate mass fraction & $x_{\mathrm{Si}}$ & $\%$ & 78.1 \\
Initial abundance of ${ }^{26} \mathrm{Al}$ & \multicolumn{1}{l}{$\left.{ }^{26} \mathrm{Al}{ }^{27} \mathrm{Al}\right]_{0}$} & - & $5.0 \times 10^{-5}$ \\
Initial abundance of ${ }^{60} \mathrm{Fe}$ & \multicolumn{1}{l}{$\left.{ }^{60} \mathrm{Fe}{ }^{56} \mathrm{Fe}\right]_{0}$} & - & $1.0 \times 10^{-8}$ \\
Average intrinsic density & $\bar{\rho}$ & $\mathrm{kg} \mathrm{m}^{-3}$ & 3740 \\
Bulk thermal conductivity & $k$ & $\mathrm{~W} \mathrm{~m}^{-1} \mathrm{~K}^{-1}$ & 4.36 \\
Specific heat capacity & $c_{\mathrm{p}}$ & $\mathrm{J} \mathrm{kg}^{-1} \mathrm{~K}^{-1}$ & $(1)$ \\
Gas constant & $\mathcal{R}$ & $\mathrm{kJ} \mathrm{mol}^{-1} \mathrm{~K}^{-1}$ & 0.00831 \\
Creep activation energy & $E$ & $\mathrm{~kJ} \mathrm{~mol}^{-1}$ & 356 \\
Grain size & $b$ & $\mathrm{~m}^{-3 / 2}$ & $10^{-6}$ \\
Pre-factor & $B$ & $\mathrm{~m}^{3} \mathrm{~Pa}^{-3 / 2} \mathrm{~s}^{-1}$ & $1.26 \times 10^{-18}$ \\
Grain size exponent & $m$ & - & 3 \\
Stress exponent & $n$ & - & $3 / 2$ \\
\hline
\end{tabular}

Notes. The density and the thermal conductivity values are stated for $\phi=0$. For the thermal conductivity of a porous assemblage the exponential fit from Krause et al. (2011) is used, which displays a variation of two orders of magnitude for porosities between any value of $\phi_{0}$ and 0 . For $E$ and $b$ the values from the table are used if not stated otherwise. For the specific heat capacity a temperature dependent fit from Yomogida \& Matsui (1984) is adopted.

References. (1) Yomogida \& Matsui (1984).

and critical porosities $\phi_{\mathrm{c}}$ (or densities $D_{\mathrm{c}}$ ) at which the geometric model is no longer valid, and different parameters to be used in Eq. (6). These parameters and the parameters that will be described in Appendix A are summarised in Table 1.

\subsection{Structural evolution of a planetesimal}

We approximate the continuous accretion of a body by considering instantaneous formation at a certain time $t_{0}$ relative to the formation of the calcium-aluminium-rich inclusions (CAIs), at which accretion is completed. The planetesimals accrete as porous aggregates and undergo compaction via hot pressing due to increasing temperature in the presence of stress. Assuming a terminal radius $\bar{R}$, which corresponds to a body with the porosity equal to zero, the initial radius $R_{\mathrm{p}}\left(t_{0}\right)$ at the accretion onset time $t_{0}$ is calculated using the prescribed uniform initial porosity $\phi_{0}$. In the case of instantaneous formation the initial radius is larger than the terminal radius and is given by

$R_{\mathrm{p}}\left(t_{0}\right)=\frac{\bar{R}}{\left(1-\phi_{0}\right)^{\frac{1}{3}}}$.

The radius changes with time as a result of sintering converging against the terminal radius $\bar{R}$ for falling average porosity $\bar{\phi}(t)$ at the time $t$ :

$R_{\mathrm{p}}(t)=\left(\frac{1-\phi_{0}}{1-\bar{\phi}(t)}\right)^{\frac{1}{3}} R_{\mathrm{p}}\left(t_{0}\right)$.

However, the terminal radius can only be reached if the body becomes completely consolidated, which is usually not the case. Thus, a planetesimal experiences a macroscopic change (shrinkage) during the microscopic change (compaction) and the depth at which a particle is located changes with the radius and the porosity distribution. 
W. Neumann et al.: Modelling of compaction in planetesimals

Table 3. Initial porosity $\phi_{0}$, initial thermal conductivity $k_{0}$, average final porosity $\bar{\phi}_{\mathrm{f}}$, initial planetesimal radius $R_{0}$, final planetesimal radius $R_{\mathrm{f}}$, thickness of the porous layer $D_{\mathrm{p}}$, central maximum temperature $T_{\max }$, and central pressure $P_{\mathrm{c}}$ at $\bar{\phi}\left(t_{0}\right)=\phi_{0}$.

\begin{tabular}{|c|c|c|c|c|c|c|}
\hline & & & SCP & ORP & RHP & $\mathrm{BCCP}$ \\
\hline & $\phi_{0}$ & $\%$ & 47.64 & 39.54 & 25.95 & 31.98 \\
\hline & $k_{0}$ & 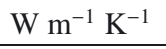 & 0.088 & 0.14 & 0.32 & 0.22 \\
\hline \multirow[t]{8}{*}{$\mathrm{S}_{\text {creep }}$} & $\bar{\phi}_{\mathrm{f}}$ & $\%$ & 31.91 & 32.64 & 25.81 & 30.55 \\
\hline & $R_{0}$ & $\mathrm{~km}$ & 6.20 & 5.91 & 5.53 & 5.69 \\
\hline & $R_{\mathrm{f}}$ & $\mathrm{km}$ & 5.68 & 5.70 & 5.52 & 5.65 \\
\hline & $D_{\mathrm{p}}$ & $\mathrm{km}$ & 2.08 & 3.25 & 5.52 & 5.65 \\
\hline & $T_{\max }$ & K & 934 & 905 & 808 & 869 \\
\hline & $P_{\mathrm{c}}$ & $\mathrm{MPa}$ & 0.021 & 0.025 & 0.033 & 0.029 \\
\hline & $I_{T}$ & K & $762-925$ & $750-905$ & $740-$ & $745-$ \\
\hline & $t_{\text {comp }}$ & Ma & 0.44 & 0.53 & 1.07 & 1.51 \\
\hline \multirow[t]{8}{*}{$\mathrm{S}_{\mathrm{par}}$} & $\bar{\phi}_{\mathrm{f}}$ & $\%$ & 16.83 & 19.10 & 21.21 & 20.89 \\
\hline & $R_{0}$ & $\mathrm{~km}$ & 6.20 & 5.91 & 5.53 & 5.69 \\
\hline & $R_{\mathrm{f}}$ & $\mathrm{km}$ & 5.32 & 5.37 & 5.41 & 5.41 \\
\hline & $D_{\mathrm{p}}$ & $\mathrm{km}$ & 1.15 & 1.59 & 3.42 & 2.30 \\
\hline & $T_{\max }$ & $\mathrm{K}$ & 734 & 727 & 706 & 717 \\
\hline & $P_{\mathrm{c}}$ & $\mathrm{MPa}$ & 0.021 & 0.025 & 0.033 & 0.029 \\
\hline & $I_{T}$ & K & $650-700$ & $650-700$ & $650-700$ & $650-700$ \\
\hline & $t_{\text {comp }}$ & $\mathrm{Ma}$ & 0.08 & 0.08 & 0.15 & 0.10 \\
\hline \multirow[t]{8}{*}{$\mathrm{B}_{\text {creep }}$} & $\bar{\phi}_{\mathrm{f}}$ & $\%$ & 25.16 & 21.15 & 15.96 & 17.72 \\
\hline & $R_{0}$ & $\mathrm{~km}$ & 62.04 & 59.13 & 55.27 & 56.85 \\
\hline & $R_{\mathrm{f}}$ & $\mathrm{km}$ & 55.07 & 54.12 & 52.98 & 53.36 \\
\hline & $D_{\mathrm{p}}$ & $\mathrm{km}$ & 22.07 & 23.70 & 25.50 & 20.30 \\
\hline & $T_{\max }$ & $\mathrm{K}$ & 739 & 739 & 738 & 739 \\
\hline & $P_{\mathrm{c}}$ & $\mathrm{MPa}$ & 2.1 & 2.5 & 3.3 & 2.9 \\
\hline & $I_{T}$ & $\mathrm{~K}$ & $662-739$ & $655-739$ & $645-738$ & $650-738$ \\
\hline & $t_{\text {comp }}$ & Ma & 9.67 & 7.89 & 6.12 & 5.28 \\
\hline \multirow[t]{8}{*}{$\mathrm{B}_{\mathrm{par}}$} & $\bar{\phi}_{\mathrm{f}}$ & $\%$ & 9.07 & 9.19 & 8.68 & 9.06 \\
\hline & $R_{0}$ & $\mathrm{~km}$ & 62.04 & 59.13 & 55.27 & 56.85 \\
\hline & $R_{\mathrm{f}}$ & $\mathrm{km}$ & 51.61 & 51.63 & 51.54 & 51.61 \\
\hline & $D_{\mathrm{p}}$ & $\mathrm{km}$ & 5.50 & 6.60 & 9.50 & 8.00 \\
\hline & $T_{\max }$ & $\mathrm{K}$ & 736 & 735 & 734 & 735 \\
\hline & $P_{\mathrm{c}}$ & $\mathrm{MPa}$ & 2.1 & 2.5 & 3.3 & 2.9 \\
\hline & $I_{T}$ & $\mathrm{~K}$ & $650-700$ & $650-700$ & $650-700$ & $650-700$ \\
\hline & $t_{\text {comp }}$ & $\mathrm{Ma}$ & 0.86 & 0.86 & 0.86 & 0.86 \\
\hline
\end{tabular}

Notes. The lower value of the temperature interval $I_{T}$ defines the temperature at which the central porosity changed by $1 \%$ of $\phi_{0}$ and the upper value defines the temperature at which $\phi=10^{-5}$ was reached. The compaction time $t_{\text {comp }}$ is the time between the onset and the end of porosity loss in the centre. Considered are bodies with terminal radii of $5 \mathrm{~km}\left(\mathrm{~S}_{\text {creep }}, \mathrm{S}_{\mathrm{par}}\right)$ and $50 \mathrm{~km}\left(\mathrm{~B}_{\text {creep }}, \mathrm{B}_{\mathrm{par}}\right)$. Thereby, compaction is modelled either via creep $\left(\mathrm{S}_{\text {creep }}, \mathrm{B}_{\text {creep }}\right)$ or as a $T$-dependent process only $\left(\mathrm{S}_{\mathrm{par}}, \mathrm{B}_{\mathrm{par}}\right)$. For each case four packings are considered with the corresponding initial porosity and radius, as well as the corresponding computation of the effective stress.

\section{Results}

Compaction of a planetesimal via hot pressing is a direct result of heating by the radioactive decay of the short-lived isotopes ${ }^{26} \mathrm{Al}$ and ${ }^{60} \mathrm{Fe}$. The initial concentrations of these isotopes in a planetesimal depend on the formation time relative to the formation of the CAIs. Because of the rapid decline in the concentration of these heat sources, a late formation time reduces the amount of heating significantly. The conductive heat loss through the surface counteracts the internal heating by radiogenic heat sources. The efficiency of this cooling depends on the surface-to-volume ratio of a body, i.e. the smaller the body, the more efficient the cooling. Another factor that influences the thermal evolution of a planetesimal is the presence of porous material which has a lower thermal conductivity compared to consolidated material and reduces conductive cooling. Assuming equal composition and material properties, a rough measure of the compaction potential of a planetesimal is the combination of its size (terminal radius $\bar{R}$; see Eq. (11)) and formation time ( $t_{0}$, relative to the formation of the CAIs). In the following we will consider two bodies for which we choose $\left(\bar{R}, t_{0}\right)=(5 \mathrm{~km}, 2.3 \mathrm{Ma})$ (case $\mathrm{S}$ refers to small body) and $\left(\bar{R}, t_{0}\right)=(50 \mathrm{~km}, 3.2 \mathrm{Ma})$ (case $\mathrm{B}$ refers to big body). For both cases, we compare the extent of compaction using either the creep-related approach described in Sect. 2 (models $\mathrm{S}_{\text {creep }}$ and $\mathrm{B}_{\text {creep }}$; see Table 3 ), or the parametrised approach, assuming linear decrease of the porosity in the temperature 

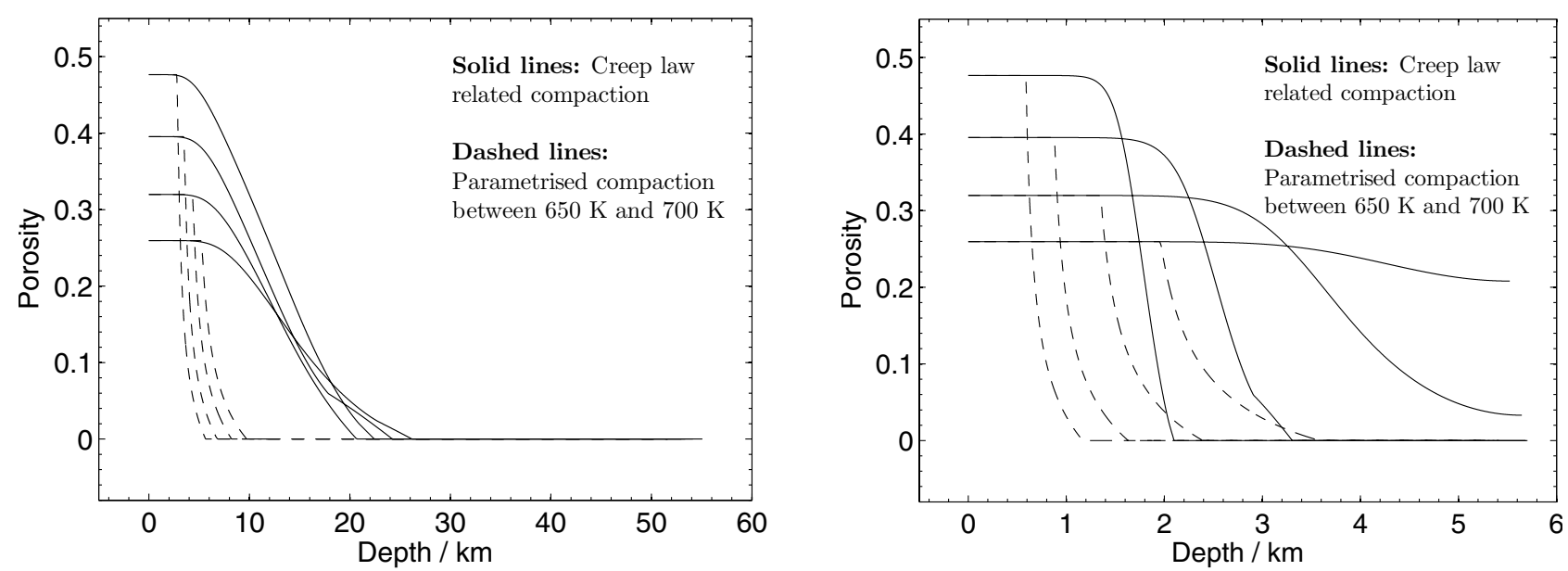

Fig. 2. Porosity after the cooling of the body below $300 \mathrm{~K}$ as a function of depth. Left panel: $\left(\bar{R}, t_{0}\right)=(50 \mathrm{~km}, 3.2 \mathrm{Ma})$, models $\mathrm{B}_{\text {creep }}$ (solid lines) and $\mathrm{B}_{\mathrm{par}}$ (dashed lines). Right panel: $\left(\bar{R}, t_{0}\right)=(5 \mathrm{~km}, 2.3 \mathrm{Ma})$, models $\mathrm{S}_{\text {creep }}$ (solid lines) and $\mathrm{S}_{\text {par }}$ (dashed lines). For each model four different packings are considered. From top to bottom (at the depth of zero km) the lines (both solid and dashed) correspond to SCP, ORP, BCCP, and RHP. We note that for the single packings the different porosities in the primordial layer result in a variation of the radius (see Table 3). In addition, for a chosen packing with different final average porosities (i.e. different thickness of the porous layer), the associated solid and dashed lines have different lengths. This is not obvious here because the dashed lines are overlaid by the solid lines at depth.

range 650-700 K (models $\mathrm{S}_{\mathrm{par}}$ and $\mathrm{B}_{\mathrm{par}}$; see Table 3). The radii were chosen in order to simulate a relatively small and a larger planetesimal, whereas the corresponding formation times were chosen such that the bodies would neither compact completely, nor would they not compact at all. The assumed composition and the physical parameters correspond to an $\mathrm{H}$ chondrite (see Table 2).

For each case, i.e. $\mathrm{S}_{\text {creep }}, \mathrm{S}_{\mathrm{par}}, \mathrm{B}_{\text {creep }}$, and $\mathrm{B}_{\mathrm{par}}$, we investigate the thermal and structural evolution assuming four different packings of dust grains with the corresponding initial porosity and radius, as well as the corresponding computation of the effective stress (for the creep related approach, see also Appendix A). All other parameters are fixed and if not stated otherwise are identical to the parameters used in Neumann et al. (2012).

We further vary the activation energy $E$ and the grain size $b$ for case $\mathrm{B}_{\text {creep}}$; both parameters strongly influence the compaction process. The activation energy is varied between $235 \mathrm{~kJ} \mathrm{~mol}^{-1}$ and $477 \mathrm{~kJ} \mathrm{~mol}^{-1}$ (see Sect. 3.2) corresponding to the measured activation energy for olivine (Schwenn \& Goetze 1978). Olivine is assumed to dominate the rheology in ordinary chondrites. As a reference value we use $E=$ $356 \mathrm{~kJ} \mathrm{~mol}^{-1}$ (Schwenn \& Goetze 1978). The grain size of the primordial material before compaction is an unknown parameter. It has been constrained by determining the size of the matrix grains in chondritic meteorites. These grains tend to be smaller than $5 \mu \mathrm{m}$ (Hutchison 2004). However, these grains may result from coarsening of smaller particles. Another constraint is provided by the interplanetary dust particles that may serve as a substitute of protoplanetary nebular particles. According to Rietmeijer (1993), the radii of interplanetary dust particles scatter from $O(1 \mathrm{~nm})$ to $O(1 \mu \mathrm{m})$, having a typical size of $0.1-0.2 \mu \mathrm{m}$. We use as a reference value a grain radius of $b=1 \mu \mathrm{m}$ in the present study, but also investigate the influence of the grain size on compaction by varying $b$ between $10^{-5} \mathrm{~m}$ and $10^{-7} \mathrm{~m}$ (see Sect. 3.3). The possibility of a non-trivial particle radius distribution that would act to reduce porosity by filling the gaps between larger grains with smaller ones is neglected here, as well as the possibility of irregular shapes, which would act to reduce the contact areas thereby increasing the porosity.
If not otherwise stated, the parameters $b=10^{-6} \mathrm{~m}$ and $E=$ $356 \mathrm{~kJ} \mathrm{~mol}^{-1}$ are used in the models.

\subsection{Parametrised vs. creep related compaction}

Figure 2 shows final porosity profiles obtained for model $\mathrm{B}_{\text {creep }}$ and $B_{\text {par }}$ (left panel), and $S_{\text {creep }}$ and $S_{\text {par }}$ (right panel). Four initial structures (packings) are considered, for which the initial (uniform) porosities vary between $26 \%$ and $47 \%$ (see Table 1 ).

The planetesimal starts as a porous body that heats up as a result of the decay of ${ }^{26} \mathrm{Al}$ and ${ }^{60} \mathrm{Fe}$. The temperature profile in such a body with a homogeneous structure and heat source distribution is monotonous with a maximum at the centre. Thus, porosity reduction starts in the central region and the compaction front proceeds from the inside outwards. Thereby, the radius and the bulk volume decrease, while the bulk density increases. Eventually, a stable porosity profile is established (see Fig. 2). The porosity as a function of radius varies nearly exponentially between 0 in the completely compacted region and the initial porosity $\phi_{0}$ in an outer layer consisting of primordial material. This insulating blanket of a variable thickness is retained at the surface, where, because of low temperature and pressure, compaction does not occur. While for decreasing porosity the heat source density increases and promotes a stronger heating, the decreasing thickness of the insulating porous layer and the shrinking radius enhance cooling of the body. The interplay of these three factors determines the temperature evolution.

For the parametrised approach of compaction (cases $S_{\text {par }}$ and $\mathrm{B}_{\mathrm{par}}$ ), both bodies consolidate completely in the centre and have a primordial porous layer of a variable thickness at the surface. The change from the consolidated region to the porous layer is very abrupt. This behaviour can be explained by the temperature profile, which is very flat apart from the porous part where the temperature decreases rapidly toward the surface. The specific shape of the profile is even more pronounced due to the insulating effect of the outer low-conductivity region. As a consequence, when the central region exceeds the temperature of $700 \mathrm{~K}$, the entire body except the outer $\lesssim 10 \mathrm{~km}$ (for $\mathrm{B}_{\text {par }}$ ) or $\$ 4 \mathrm{~km}$ (for $\mathrm{S}_{\mathrm{par}}$ ) experiences similar heating and compacts to $\phi=0$, the porous blanket $D_{\mathrm{p}}$ being rather thin $(5.5-9.5 \mathrm{~km}$ 
for $\mathrm{B}_{\mathrm{par}}$ and 1.1-3.4 $\mathrm{km}$ for $\mathrm{S}_{\mathrm{par}}$; see Table 3). Using a different interval for sintering, e.g. $670-700 \mathrm{~K}$, the same scenario would apply in general. The porous layer will be slightly thicker and the porosity profile will have a steeper slope between the compacted and porous regions. The lack of strong insulation and the decline of the heat production inhibit further temperature increase. Thus, for $\mathrm{S}_{\mathrm{par}}$ and $\mathrm{B}_{\mathrm{par}}, T_{\max }$ varies between 706-734 K and $734-736 \mathrm{~K}$, respectively, and remains below $800 \mathrm{~K}$ in all cases.

In contrast, for the creep related approach, the porosity changes nearly exponentially with time and temperature. Compaction of the planetesimal is less efficient because a temperature of $700 \mathrm{~K}$ is not sufficient to achieve $\phi=0$ even for the larger body with a reference radius of $\bar{R}=50 \mathrm{~km}$. Thus, the low conductivity of the porous material provokes further heating of the interior, until temperatures of up to $925 \mathrm{~K}$ (case $\mathrm{S}_{\text {creep }}$ ) or only up to $739 \mathrm{~K}$ (case $\mathrm{B}_{\text {creep }}$ ) are reached. Even for those temperatures, the porosity reduction is either moderate (case $\mathrm{B}_{\text {creep }}$ ), or nearly non-existent (case $S_{\text {creep }}$, the profile corresponding to the packing RHP in Fig. 2). The outer porous layer remains significantly thicker than for the parametrised approach with values between 20-25 $\mathrm{km}$ for $\mathrm{B}_{\text {creep }}$ and more than $2 \mathrm{~km}$ (or even comprising the entire body) for $S_{\text {creep }}$. As a consequence, using the creep related approach for hot pressing results in much higher average porosities after compaction in contrast to the parametrised approach. For case $S_{\text {creep }}$, the average porosity is a factor of 1.2-1.9 larger than for $S_{\text {par }}$, and for case $B_{\text {creep }}$ it is a factor of 1.8-2.8 larger than for $\mathrm{B}_{\mathrm{par}}$ (we note that different factors correspond to different packings).

It should also be noted that the choice of the packing has a larger influence on small bodies with low pressure than on larger bodies with higher pressure. This can be seen in the final structure (Fig. 2 and Table 3) and therefore also has a consequence on the temperature evolution. The maximum temperatures vary by less than $40 \mathrm{~K}$ for different packings in the case of $S_{\text {par }}, B_{\text {creep }}$, and $B_{p a r}$, but for $S_{\text {creep }}$ variation of the maximum temperature reaches $120 \mathrm{~K}$. This finding is the result of the low pressure in small bodies, resulting in a strong variation of the porosity profiles for different packings.

Table 3 lists initial and final properties for all cases. The parameter $I_{T}$ defines the temperature interval of compaction in the centre of the body under consideration. For creep-related models, the lower value of $I_{T}$ corresponds to the temperature at which the porosity changes by $1 \%$ of its initial value $\phi_{0}$, while the upper value corresponds to the temperature at which the porosity decreases below $10^{-5}$. For parametrised models, the lower and upper values of $I_{T}$ indicate the temperatures at which compaction starts and is completed, respectively (by definition, 650-700 K). If only one value is given, then the centre did not compact completely. In general, for the large body case (i.e. $\mathrm{B}_{\text {creep }}$ with an initial central pressure of 2.1-3.3 $\mathrm{MPa}$ depending on the value of $\left.\phi_{0}\right), I_{T}$ varies around $650-740 \mathrm{~K}$. For the small body case (i.e. $\mathrm{S}_{\text {creep }}$ with an initial central pressure of $0.021-0.033 \mathrm{MPa}$ ), the interval $I_{T}$ varies around $740-925 \mathrm{~K}$ and for the packings RHP and BCCP, compaction in the centre stops even before reaching $\phi=0$. In the latter cases, the maximum temperature of $808 \mathrm{~K}$ or $869 \mathrm{~K}$ were not sufficiently high to reach $\phi=0$. It should be noted that $I_{T}$ has been constrained for the centre of the respective body and the differences in $I_{T}$ between the parametrised and the creep related cases are even larger in the more shallow regions.

To some extent, the timescale of the compaction can be estimated by using the relation $t_{\text {comp }} \approx \eta \sigma_{1}^{-3 / 2}$, where $\eta=B^{-1} b^{3} \mathrm{e}^{\frac{E}{R T}}$

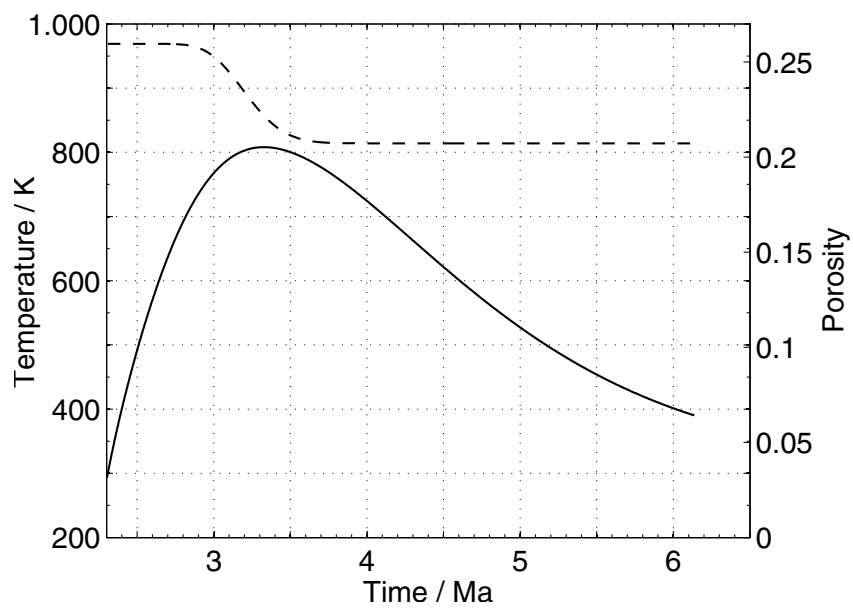

Fig. 3. Central temperature (solid line) and central porosity (dashed line) as functions of time for the case $\mathrm{S}_{\text {creep }}$ with RHP.

(i.e. by inverting Eq. (9)). In the following we demonstrate this in two examples: $B_{\text {creep }}$ with ORP (see Fig. 6, left panel) and $S_{\text {creep }}$ with RHP (see Fig. 3).

$B_{\text {creep }}$ with $O R P$ : the central porosity changes by $1 \%$ of its initial value of $\phi_{0}=0.3954$ at the temperature of $655 \mathrm{~K}$ (see Table 3 ). The central pressure $\sigma_{0}$ at this time is $2.5 \mathrm{MPa}$. At the relative density of $\approx 0.61$, the effective stress is $\sigma_{1} \approx 200 \sigma_{0} \approx 500 \mathrm{MPa}$. For this effective stress and for the temperature of $T=655 \mathrm{~K}$, we obtain $t_{\text {comp }} \approx 52 \mathrm{Ma}$. Subsequently, the temperature increases, while the effective stress and the porosity decrease. At $t \approx 6.6 \mathrm{Ma}$ and $T \approx 722 \mathrm{~K}$ the values of $\phi \approx 0.3, \sigma_{1} \approx 10 \sigma_{0}$ and $\sigma_{0} \approx 2.9 \mathrm{MPa}$ follow. Thus, $t_{\text {comp }}$ is reduced to $8.6 \mathrm{Ma}$. In fact, the pores around the centre close at $t=12.8 \mathrm{Ma} \leq$ $6.6 \mathrm{Ma}+8.6 \mathrm{Ma}=15.2 \mathrm{Ma}$ predicted by the last estimate (see Fig. 6; we note that the temperature increases further up to $739 \mathrm{~K})$.

$S_{\text {creep }}$ with RHP: the central porosity changes by $1 \%$ of its initial value of $\phi_{0}=0.2595$ at the temperature of $740 \mathrm{~K}$ (see Table 3). The central pressure $\sigma_{0}$ at this time is $0.033 \mathrm{MPa}$. At the relative density of $\approx 0.7431$, the effective stress is $\sigma_{1} \approx 100 \sigma_{0} \approx$ 3.3 MPa. For this effective stress and for the temperature of $T=740 \mathrm{~K}$, we obtain $t_{\text {comp }} \approx 53 \mathrm{Ma}$. Subsequently, the temperature increases, while the effective stress and the porosity decrease. At $t \approx 3.34 \mathrm{Ma}$ and $T \approx 808 \mathrm{~K}$ the values of $\phi \approx 0.22$, $\sigma_{1} \approx 13 \sigma_{0}$, and $\sigma_{0} \approx 0.0334 \mathrm{MPa}$ follow. Thus, $t_{\text {comp }}$ is reduced to $8.6 \mathrm{Ma}$. However, the temperature falls right after reaching $808 \mathrm{~K}$ rapidly (see Fig. 3 below) and compaction stalls.

\subsection{Variation of the activation energy}

In the following, we vary the creep activation energy for model $\mathrm{B}_{\text {creep }}$ within the interval $E=356 \pm 121 \mathrm{~kJ} \mathrm{~mol}^{-1}$ (Schwenn \& Goetze 1978). The general compaction scenario is identical to the one described above. Figure 4 shows the final porosity profiles and Table 4 lists the final properties of the planetesimal obtained for different values of the activation energy. The final average porosity is $\approx 3 \%$ for $E=235 \mathrm{~kJ} \mathrm{~mol}^{-1}$ and increases monotonously with increasing value of $E$. For the maximum value of $E=477 \mathrm{~kJ} \mathrm{~mol}^{-1}$ compaction is almost negligible and the final porosity remains $\approx \phi_{0}$. Although the interior structure differs significantly due to a variation in $E$, the maximum temperature in the centre increases only moderately with increasing $E$ by $\approx 2 \%$ from $727 \mathrm{~K}$ to $740 \mathrm{~K}$. This is caused by 


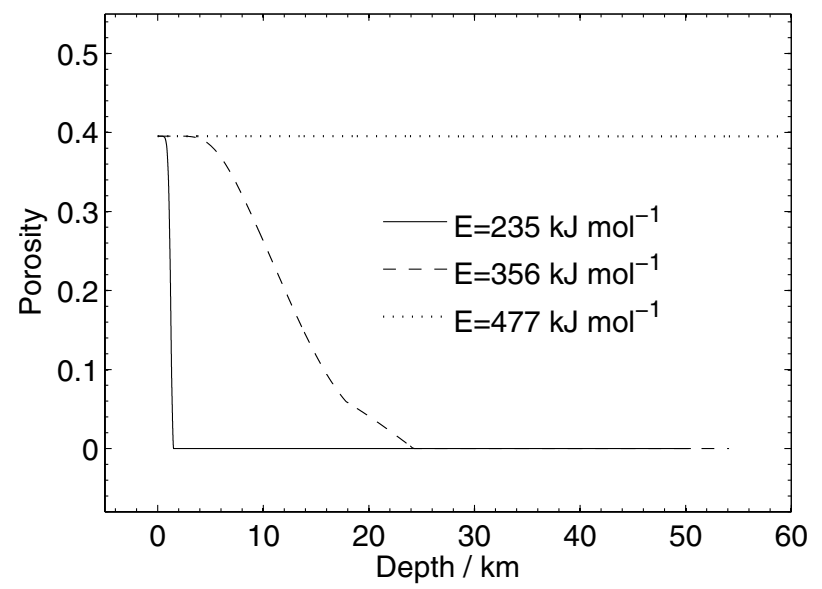

Fig. 4. Porosity as a function of depth for $\left(\bar{R}, t_{0}\right)=(50 \mathrm{~km}, 3.2 \mathrm{Ma})$ for model $\mathrm{B}_{\text {creep }}$ assuming ORP. The lines correspond to the adopted values of the activation energy $E$ within the interval inferred by Schwenn \& Goetze (1978).

Table 4. Average final porosity $\bar{\phi}_{\mathrm{f}}$, final planetesimal radius $R_{\mathrm{f}}$, thickness of the porous layer $D_{\mathrm{p}}$, central maximum temperature $T_{\max }$, and the temperature interval $I_{T}$ in which compaction took place in the centre (see also Table 3).

\begin{tabular}{llccc}
\hline \hline$E$ & $\mathrm{~kJ} \mathrm{~mol}^{-1}$ & 235 & 356 & 477 \\
\hline $\bar{\phi}_{\mathrm{f}}$ & $\%$ & 2.95 & 21.15 & 39.50 \\
$R_{\mathrm{f}}$ & $\mathrm{km}$ & 50.50 & 54.12 & 59.13 \\
$D_{\mathrm{p}}$ & $\mathrm{km}$ & 1.50 & 23.70 & 59.13 \\
$T_{\max }$ & $\mathrm{K}$ & 727 & 739 & 740 \\
$I_{T}$ & $\mathrm{~K}$ & $442-529$ & $655-739$ & - \\
\hline
\end{tabular}

Notes. Considered is the body $\left(\bar{R}, t_{0}\right)=(50 \mathrm{~km}, 3.2 \mathrm{Ma})$ with the model $\mathrm{B}_{\text {creep }}$. The activation energy for creep is varied between the values of $235 \mathrm{~kJ} \mathrm{~mol}^{-1}$ and $477 \mathrm{~kJ} \mathrm{~mol}^{-1}$. All other parameters are fixed and correspond to the case $\mathrm{B}_{\text {creep }}$ assuming ORP.

the decline in the heat production with time. Even if the interior remains more porous with increasing $E$ and could heat up efficiently because of its low thermal conductivity, not enough energy is available to strongly heat the interior. Smaller activation energy implies compaction onset at a lower temperature and a shift of $I_{T}$ to lower temperatures. Consequently, a larger part of the interior consolidates completely, and the thickness of the porous layer is smaller than for higher values of $E$, which also implies a weaker isolation and stronger cooling leading to a slightly lower $T_{\max }$. The interval $I_{T}$ experiences a considerable shift. While for $E=235 \mathrm{~kJ} \mathrm{~mol}^{-1}$ compaction in the centre takes place at $T \geq 440 \mathrm{~K}$, for $E=356 \mathrm{~kJ} \mathrm{~mol}^{-1}$ it takes place at $T \geq 650$. For the extreme case of $E=477 \mathrm{~kJ} \mathrm{~mol}^{-1}$ the threshold temperature for compaction has not been reached and thus the porosity change is infinitesimal.

\subsection{Variation of the grain size}

In this section we address the influence of the grain size while keeping all other parameters fixed. Figure 5 shows the final porosity profiles. The other output parameters such as average final porosity $\bar{\phi}_{\mathrm{f}}$, final planetesimal radius $R_{\mathrm{f}}$, thickness of the porous layer $D_{\mathrm{p}}$, central maximum temperature $T_{\max }$, and the temperature interval $I_{T}$ in which compaction took place in the centre, are listed in Table 5. All parameters are identical to those used for the case $\mathrm{B}_{\text {creep }}$ with ORP, except the grain size $b$.

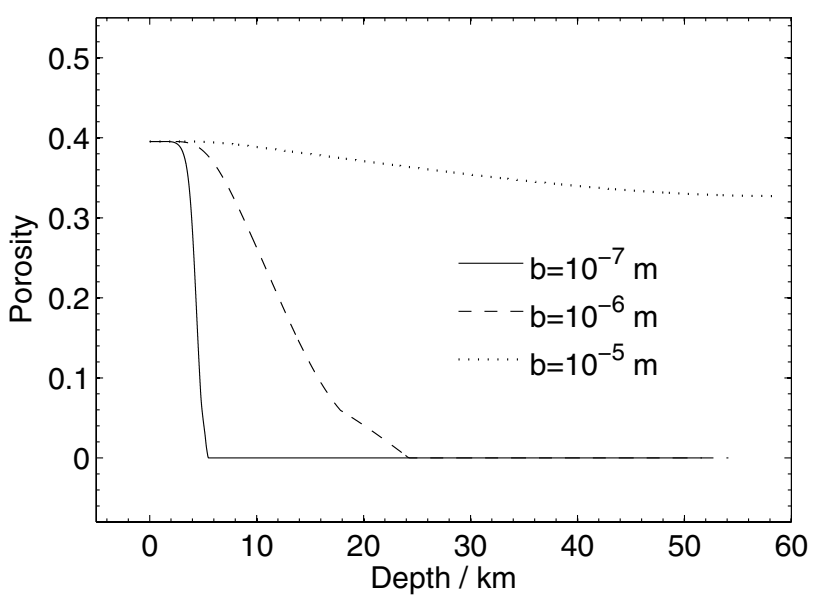

Fig. 5. Porosity as a function of depth for $\left(\bar{R}, t_{0}\right)=(50 \mathrm{~km}, 3.2 \mathrm{Ma})$ for model $\mathrm{B}_{\text {creep }}$ assuming ORP. The lines correspond to the adopted values of the grain size $b$. The grain size varies by two orders of magnitude between $b=10^{-7}$ and $b=10^{-5} \mathrm{~m}$.

Table 5. Average final porosity $\bar{\phi}_{\mathrm{f}}$, final planetesimal radius $R_{\mathrm{f}}$, thickness of the porous layer $D_{\mathrm{p}}$, central maximum temperature $T_{\max }$, and the temperature interval $I_{T}$ in which compaction took place in the centre (see also Table 3).

\begin{tabular}{llccc}
\hline \hline$b$ & $\mathrm{~m}$ & $10^{-5}$ & $10^{-6}$ & $10^{-7}$ \\
\hline $\bar{\phi}_{\mathrm{f}}$ & $\%$ & 37.92 & 21.15 & 9.23 \\
$R_{\mathrm{f}}$ & $\mathrm{km}$ & 58.61 & 54.12 & 51.64 \\
$D_{\mathrm{p}}$ & $\mathrm{km}$ & 58.61 & 23.70 & 5.40 \\
$T_{\max }$ & $\mathrm{K}$ & 740 & 739 & 736 \\
$I_{T}$ & $\mathrm{~K}$ & $716-$ & $655-739$ & $597-694$ \\
\hline
\end{tabular}

Notes. Considered is the body $\left(\bar{R}, t_{0}\right)=(50 \mathrm{~km}, 3.2 \mathrm{Ma})$ with the model $\mathrm{B}_{\text {creep. }}$. The grain size varies between the values of $10^{-5} \mathrm{~m}$ and $10^{-7} \mathrm{~m}$. All other parameters are fixed and correspond to the case $\mathrm{B}_{\text {creep }}$ assuming ORP.

There is a straightforward relationship between the adopted value of the grain radius and the extent of compaction. For decreasing $b$ the final average porosity and the maximum temperature decrease as well. Assuming $b=10^{-5} \mathrm{~m}$ (i.e. $b=10 \mu \mathrm{m}$ ), the porosity starts decreasing at a temperature of $716 \mathrm{~K}$, but as the maximum temperature is only $740 \mathrm{~K}$, compaction stops at $\phi=0.33$ in the centre. For the reference case with $b=10^{-6} \mathrm{~m}$, a substantial part of the interior becomes completely consolidated. With decreasing grain size, i.e. $b=10^{-7} \mathrm{~m}$ for the case $\mathrm{B}_{\text {creep }}$, compaction is even more effective, taking place between $597 \mathrm{~K}$ and $694 \mathrm{~K}$ in the centre. Although the temperature interval $I_{T}$ for $b=10^{-6} \mathrm{~m}$ is closer to $650-700 \mathrm{~K}$, at smaller depths the interval changes towards higher temperatures. Eventually, a porous outer layer with a thickness of only $\approx 5 \mathrm{~km}$ is established for $b=10^{-7} \mathrm{~m}$. Similarly to the effect of the activation energy, a smaller value of $b$ favours creep at lower temperatures. This leads to a thinner insulating layer and better cooling, resulting in lower temperatures and thus lower $T_{\max }$.

In conclusion, it is easier to achieve consolidation of a finegrained material via hot pressing than for coarse-grained material. For the latter, higher temperatures than those that are possible for the pair $\left(\bar{R}, t_{0}\right)=(50 \mathrm{~km}, 3.2 \mathrm{Ma})$ are needed to change the porosity further (e.g. through an earlier formation time). We have explored this aspect with some test runs by increasing the grain radius even further up to the size of $1 \mathrm{~cm}$ appropriate for 

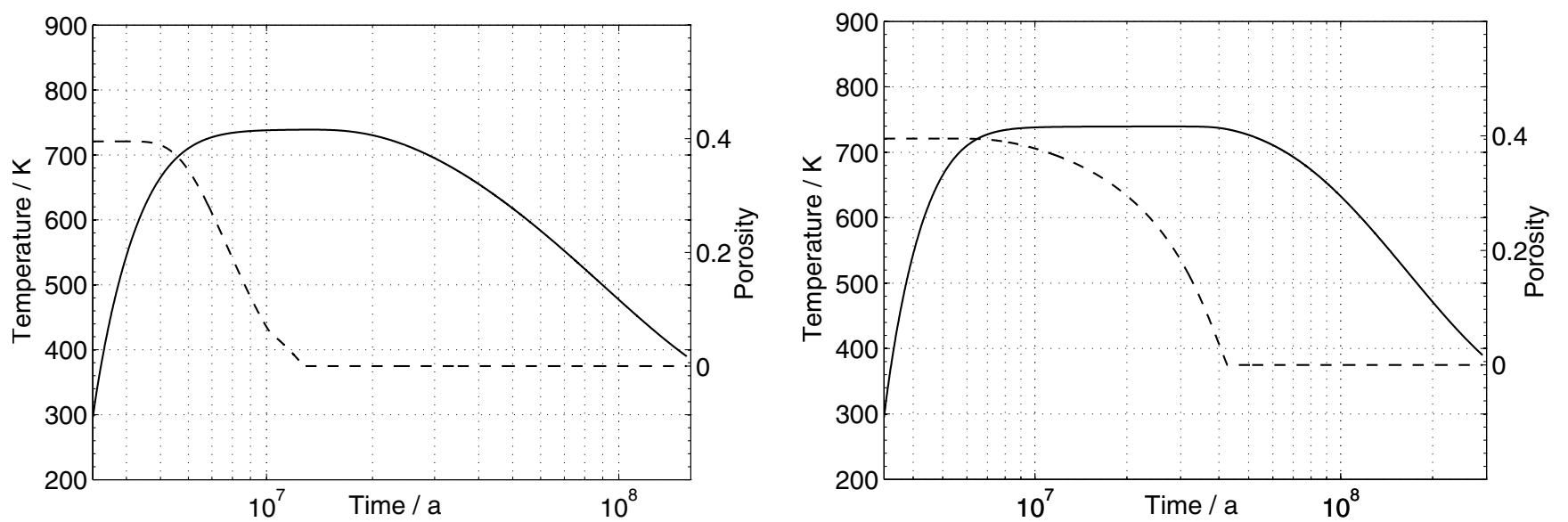

Fig. 6. The central temperature (solid lines) and the central porosity (dashed lines) as functions of time. Left panel: case $\left(\bar{R}, t_{0}\right)=(50 \mathrm{~km}, 3.2 \mathrm{Ma})$ with ORP and the associated effective stress $\sigma_{1}$. Right panel: same as left panel but lithostatic pressure $\sigma_{0}$ used instead of the effective stress $\sigma_{1}$.

chondrules (Weisberg et al. 2006). For that extreme case, some residual porosity was present even at super-solidus temperatures of the metal phase and even of the silicate phase $(T \geq 1425 \mathrm{~K})$ of ordinary chondritic material. This means that at moderate pressures and for certain values of activation energy, compaction takes place simultaneously with the production of partial melt (see Discussion).

\subsection{Effective stress}

The compaction model used considers that for porous material the effective stress can be several orders of magnitude higher than the stress caused simply by lithostatic pressure (see Fig. 2). In Fig. 6 we show the influence of the efffective stress on the evolution of the central temperature (solid lines) and of the central porosity (dashed lines). The left panel corresponds to the case $\left(\bar{R}, t_{0}\right)=(50 \mathrm{~km}, 3.2 \mathrm{Ma})$ with ORP and the associated effective stress $\sigma_{1}$, whereas the right panel corresponds to a simulation where the lithostatic pressure was used instead of the effective stress. While in the first case the central porosity changes by $1 \%$ of its initial value $\phi_{0}$ at the temperature of $655 \mathrm{~K}$ and reaches zero at $739 \mathrm{~K}$ (see Table 3), in the second case compaction requires a higher threshold temperature and starts at $732 \mathrm{~K}$. Compaction is, however, completed for $739 \mathrm{~K}$ as in the case using the effective stresses. Additional output parameters of this simulation are $\bar{\phi}_{\mathrm{f}}=34.40 \%, R_{\mathrm{f}}=57.57 \mathrm{~km}$, $D_{\mathrm{p}}=41.20 \mathrm{~km}$, and $T_{\max }=739 \mathrm{~K}$ (for the same case with a proper $\sigma_{1}$ we had $\bar{\phi}_{\mathrm{f}}=21.15 \%, R_{\mathrm{f}}=54.12 \mathrm{~km}, D_{\mathrm{p}}=23.70 \mathrm{~km}$, and $T_{\max }=739 \mathrm{~K}$; see Table 3 ). Although the maximum temperature is the same, the smaller values of $\sigma_{0}$ compared to those of $\sigma_{1}$ (see Fig. 1) lead to a less effective compaction. Thus, the insulation of the centre is more effective $\left(D_{\mathrm{p}}=41.20 \mathrm{~km}\right)$ and the temperature of $\geq 700 \mathrm{~K}$ in the centre can be maintained for more than $60 \mathrm{Ma}$. This allows the centre to compact to $\phi=0$ after $\approx 40 \mathrm{Ma}$ (in the standard case with $\sigma_{1}$ only $\approx 8$ Ma were needed, compare the compaction time $t_{\text {comp }}$ in Table 3 ).

The compaction timescale can be roughly estimated as in 3.1. If not the effective stress, but the central lithostatic pressure is used, for $T=739 \mathrm{~K}$ and $\sigma_{0}=2.5 \mathrm{MPa}$ we obtain $t_{\text {comp }} \approx 87 \mathrm{Ma}$. However, the pressure increases upon compaction up to the value of $4.6 \mathrm{MPa}$. For this value, $t_{\mathrm{comp}} \approx 35 \mathrm{Ma}$ follows. This agrees very well with the results obtained from the simulations.
The porosity and temperature in Fig. 6 show that compaction by hot pressing is influenced strongly by the interplay of the temperature and the applied stresses and their temporal evolution. A lack of pressure can be compensated by heating far above some critical temperature (onset of compaction at $732 \mathrm{~K}$ ). Furthermore, although for $t_{0}=3.2 \mathrm{Ma}$ a higher maximum temperature than $739 \mathrm{~K}$ cannot be reached, a drastically prolonged heating of the centre due to an effective insulation by the porous shell is possible, leading eventually to the closure of the pores.

\section{Summary and discussion}

Here we compare hot pressing via the creep related approach (Kakar \& Chaklader 1967; Rao \& Chaklader 1972; Schwenn \& Goetze 1978) with the simplified parametrised approach used by several studies (e.g. Hevey \& Sanders 2006; Sahijpal et al. 2007; Gupta \& Sahijpal 2010; Solano et al. 2012). Using the creep related approach, hot pressing is triggered when a powder assemblage reaches a certain temperature. This critical (or threshold) temperature is a function of the effective stress for otherwise fixed parameters. The higher the effective stress, the lower the critical temperature. Thus, the threshold temperature varies with depth (pressure) within a planetesimal. Since compaction is a creep process, to fully compact the material, this threshold temperature must either be sustained for a sufficiently long time, or higher temperatures are necessary to enhance the compaction rate. Thus, compaction in a thermally evolving planetesimal takes place in a certain temperature interval for a given stress. In our study, we found that for a central pressure of 200-300 MPa (equivalent to a planetesimal with a radius of $500 \mathrm{~km}$ ) the temperature interval is $597-690 \mathrm{~K}$, for a pressure of $2-3 \mathrm{MPa}$ (equivalent to a planetesimal with a radius of $50 \mathrm{~km}$ ) it is $645-739 \mathrm{~K}$, and for a pressure of $0.02-0.03 \mathrm{MPa}$ (equivalent to a planetesimal with a radius of $5 \mathrm{~km}$ ) it is 740-925 K, assuming an activation energy of $356 \mathrm{~kJ} \mathrm{~mol}^{-1}$ (Table 2).

In conclusion, only for a rather narrow parameter space or pressure range in a planetesimal, compaction occurs approximately between $650 \mathrm{~K}$ and $700 \mathrm{~K}$ as used in the parametrised approach. Depending on the pressure, initial grain size, activation energy and initial porosity, the temperature interval is instead $500-1000 \mathrm{~K}$. For some extreme values of $E$ and $b$, the upper boundary exceeds even the solidus temperature of the silicates. Significant differences in the interior structure and temperature 
evolution are the consequence; with the simplified approach compaction is overestimated predicting final porosities that are too low and thus a cooling that is unrealistically fast.

For the small body of $5 \mathrm{~km}$ radius and a formation time $t_{0}$ of $2.3 \mathrm{Ma}$ the porous layer is at least $1 \mathrm{~km}$ thinner and for a body of $50 \mathrm{~km}$ radius and $t_{0}=3.2 \mathrm{Ma}$ at least $12 \mathrm{~km}$, if the parametrised approach is used. Differences in the maximum temperatures between the two approaches can be observed, especially for small bodies (radius $\approx O(1 \mathrm{~km})$ ). The maximum temperature varies by up to $32 \%$ as the consequence of different extents of compaction.

We note that although porosity plays a substantial role in the thermal evolution and interior structure of undifferentiated planetesimals, in the case of high levels of partial melt and efficient differentiation, effects associated with porosity and compaction would be painted over in the subsequent evolution.

The parameter that has the strogest influence is the activation energy $E$ of the material. A small value of $E$ shifts the onset of compaction to lower temperatures, and a high $E$ to higher temperatures. For our cases with $E=477 \mathrm{~kJ} \mathrm{~mol}^{-1}$, some left-over porosity is observed in the centre directly before the melting of the metal-rich phase begins. This in fact requires a simultaneous treatment of porosity loss and melting. Experiments by e.g. Kohlstedt (2002) and Jin et al. (1994) suggest that a few percent of melt can reduce the creep strength by more than one order of magnitude because of the fast diffusion of the melt (see also Karato 2013). Therefore, it is likely that in these specific cases partial melting provokes quasi-instantaneous compaction on a geological timescale.

The activation energy is in general related to the mineralogical composition. For planetesimals with a "wet" rheology, i.e. containing hydrated silicates or ice, as suggested for carbonaceous chondritic compositions that are dominated by serpentine, the activation energy is lower than for dry material and thus lower compared to the ordinary chondritic composition assumed in the current study (Hilairet et al. 2007). For the ordinary chondritic composition it is assumed that olivine dominates the rheology. On the other hand, the creep activation energy of an enstatite chondritic composition seems to be higher than that of the ordinary chondritic composition (Karato 2013). This would also suggest that a carbonaceous chondritic body will compact at lower temperatures than the ordinary chondritic and the enstatitic ones at similar pressure. It should be noted, however, that the pre-factor $B$, the stress exponent $n$, and the grain size exponent $m$ need to be adjusted to the rheological properties of the material.

The shift of the compaction temperature interval to lower temperatures in a body consisting of a carbonaceous chondritic assemblage also implies that compaction and dehydration of hydrated minerals ( $\approx 298-1073 \mathrm{~K}$, Castillo-Rogez 2011) occur simultaneously. The shrinkage due to hot pressing overlaps with the shrinkage resulting from the density changes of the silicate minerals due to the dehydration on the one hand and by the filling of the pores with the water freed by the dehydration process on the other hand. Furthermore, dehydration of certain minerals proceeds in some cases simultaneously with the hydration of other silicate minerals, which could be accompanied by expansion. Thus, compaction of planetesimals that contained water in some form is an even more complex issue than compaction of rocky bodies.

Four different packings have been compared on the compaction process. The assumption on the packing of the dust grains influences the initial porosity and is particularly important for small bodies with $\bar{R}=O(1 \mathrm{~km})$; a variation in $T_{\max }$ by up to $16 \%$ has been observed. With increasing body radii the offset becomes gradually smaller and for a planetesimal radius of $50 \mathrm{~km}$ and larger the choice of the suitable packing of grains is less important.

We have further examined the influence of the effective stress on the compaction history. The results indicate that neglecting the effective stress, which acts on the contact points of the grains in case of porous material, and assuming only the lithostatic pressure leads to a longer timescale of compaction and to a higher threshold temperature at which compaction starts. Thus, compaction is underestimated when neglecting the effective stress. This finding might have implications for a number of studies that calculate or estimate the porosity reduction of a porous layer and only consider the lithostatic pressure for compaction (e.g. Wieczorek et al. 2013; Nimmo et al. 2003; Fu \& Elkins-Tanton 2014). The timescale of the porosity loss $t_{\text {comp }}$ varies in our calculations between $O(0.1 \mathrm{Ma}$ ) and $O(10 \mathrm{Ma}$ ) (see Table 3), which argues against assumptions of fixed compaction timescales utilised in some planetesimal studies.

In the current approach, we neglect compaction prior to hot pressing and assume that the initial porosity $\phi_{0}$ of the material is limited by the porosities of the random close and random loose packing. The initial porosity, however, may be reduced by a purely mechanical mechanism called cold pressing, which is driven by the applied pressure. Cold pressing does not impose any further requirements and can act at any temperature. Porosity evolution due to cold pressing is an important topic in the study of dust coagulation and planetesimal formation in protoplanetary discs and was studied in theory in e.g. Ormel et al. (2007), Suyama et al. (2008), Zsom et al. (2010, 2011), Kataoka et al. (2013a). This kind of compaction was also discussed and investigated through the laboratory experiments of Guettler et al. (2009) and the numerical experiments of Kataoka et al. (2013b). The latter authors derived a relation for the change of the relative density $D$ with the applied stress $\sigma_{0}$. In the limit $\sigma_{0} \rightarrow \infty$, the maximum relative density of $D_{\max }=0.58$ (or minimal porosity $\phi_{\min }^{\text {cold }}=0.42$ is obtained. In conclusion, it is only possible to compact porous material by cold pressing to the limit of $\phi=0.42$. Since SCP with $\phi_{0}=0.4764$ is the only model with an initial porosity of $\phi_{0} \geq 0.4$, we neglect this kind of compaction in the present study.

As a further complication, however, recent dynamic cold pressing experiments of Beitz et al. (2013) indicate that this process might have been important during accretion. Beitz et al. (2013) used aluminium cylinders as projectiles to compact the chondrite-analog samples with the initial porosities of 39-70\% in a velocity range of $1200-165 \mathrm{~m} \mathrm{~s}^{-1}$, down to the porosities of $1-30 \%$. Taking the projectile velocity as velocity of the accreting dust particles suggests that a smaller initial porosity $\phi_{0}$ should be used than the values corresponding to the packings. Moreover, this cold pressing experiment does most certainly result in crushing of the dust grains, meaning that the geometric model for the computation of the effective stress would be not applicable without further adjustments. Here future studies are needed in order to assess how this might change compaction and the structural and thermal evolution of a planetesimal.

Acknowledgements. We thank the reviewer Satoshi Okuzumi for his constructive and helpful suggestions and comments on the paper. This work was supported by the Deutsche Forschungsgemeinschaft (DFG) Priority Programme 1385 "The First 10 Million Years of the Solar System - a Planetary Materials Approach" and by the Helmholtz Association through the research alliance "Planetary Evolution and Life". 
W. Neumann et al.: Modelling of compaction in planetesimals

\section{References}

Akridge, G., Benoit, P. H., \& Sears, D. W. G. 1997, Lunar and Planetary Science Conf., XXVIII, 1178

Akridge, G., Benoit, P. H., \& Sears, D. W. G. 1998, Icarus, 132, 185

Beitz, E., Guettler, C., Nakamura, A. M., Tsuchiyama, A., \& Blum, J. 2013, Icarus, 225, 558

Bystricky, M., Lawlis, J., Mackwell, S. J., Heidelbach, F., \& Raterron, P. C. 2011, in American Geophysical Union, Fall Meeting 2011, T13A-2366

Castillo-Rogez, J. C. 2011, Icarus, 215, 599

Coogan, A. H., \& Manus, R. W. 1975, Compaction of Coarse-Grained Sediments, I, eds. G. V. Chilingarian, \& K. H. Wolf (Elsevier Scientific Publishing Company), 78

Dullien, F. A. L. 1992, Porous Media. Fluid Transport and Pore Structure, 2nd edn. (Academic Press)

Durham, W. B., Stern, L. A., \& Kirby, S. H. 2001, J. Geophys. Res., 106, 11031

Elkins-Tanton, L. T., Weiss, B. P., \& Zuber, M. T. 2011, Earth and Planetary Science Letters, 305, 1

Farnsworth, P. L., \& Coble, R. L. 1966, J. Amer. Ceramic Soc., 49, 264

Fowler, A. C. 1985, Geophys. Astrophys. Fluid Dyn., 33, 63

Fu, R. R., \& Elkins-Tanton, L. T. 2014, Earth Planet. Sci. Lett., 390, 128

Goldsby, D. L., \& Kohlstedt, D. L. 2001, J. Geophys. Res., 106, 11017

Guettler, C., Krause, M., Geretshauser, R., Speith, R., \& Blum, J. 2009, ApJ, 701,130

Gupta, G., \& Sahijpal, S. 2010, J. Geophys. Res., 115, E08001

Henke, S., Gail, H.-P., Trieloff, M., Schwarz, W. H., \& Kleine, T. 2012, A\&A, 537, A45

Hevey, P., \& Sanders, I. 2006, Meteoritics, 41, 95

Hilairet, N., Reynard, B., Wang, Y., et al. 2007, Science, 318, 1910

Hutchison, R. 2004, Meteorites: A petrologic, chemical and isotopic synthesis (Cambridge University Press)

Jin, Z. M., Green, H. W., I., \& Zhou, Y. 1994, Nature, 372, 164
Kakar, A. K., \& Chaklader, A. C. D. 1967, J. Appl. Phys., 38, 3223

Karato, S.-I. 2013, Physics and Chemistry of the Deep Earth (Wiley)

Kataoka, A., Tanaka, H., Okuzumi, S., \& Wada, K. 2013a, A\&A, 557, L4

Kataoka, A., Tanaka, H., Okuzumi, S., \& Wada, K. 2013b, A\&A, 554, A4

Kohlstedt, D. 2002, Plastic Deformation of Minerals and Rocks, eds. S. Karato,

\& H.-R. Wenk (Washington: Mineralogical Society of America), 121

Krause, M., Henke, S., Gail, H.-P., et al. 2011, Lunar and Planeteary Science Conf. Letters, 42, 2696

Moskovitz, N., \& Gaidos, E. 2011, Meteoritics, 46, 903

Neumann, W., Breuer, D., \& Spohn, T. 2012, A\&A, 543, A141

Neumann, W., Breuer, D., \& Spohn, T. 2013, Icarus, 224, 126

Nimmo, F., Pappalardo, R. T., \& Giese, B. 2003, Icarus, 166, 21

Ormel, C. W., Spaans, M., \& Tielens, A. G. G. M. 2007, A\&A, 461, 215

Rao, A. S., \& Chaklader, A. C. D. 1972, J. Amer. Ceramic Soc., 55, 596

Rietmeijer, F. J. M. 1993, Earth Planet. Sci. Lett., 117, 609

Sahijpal, S., Soni, P., \& Gupta, G. 2007, Meteoritics, 42, 1529

Schwenn, M. B., \& Goetze, C. 1978, Tectonophysics, 48, 41

Senshu, H. 2006, Frontier Research on Earth Evolution, 2, 5

Solano, J. M., Kiefer, W. S., \& Mittlefehldt, D. W. 2012, in Lunar and Planetary Science, XLIII, 2063

Suyama, T., Wada, K., \& Tanaka, H. 2008, ApJ, 684, 1310

Weisberg, M. K., McCoy, T. J., \& Krot, A. N. 2006, Systematics and Evaluation of Meteorite Classification. In: Meteorites and the Early Solar System II, 19, eds. D. S. Lauretta, \& H. Y. McSwen (Tucson: University of Arizona Press)

Wieczorek, M. A., Neumann, G. A., Nimmo, F., et al. 2013, Science, 339, 671

Wilkinson, S. L., McCoy, T. J., McCamant, J. E., Robinson, M. S., \& Britt, D. T. 2003, Meteoritics, 38, 1533

Yomogida, K., \& Matsui, T. 1984, Earth Planet. Sci. Lett., 68, 34

Zsom, A., Ormel, C. W., Güttler, C., Blum, J., \& Dullemond, C. P. 2010, A\&A, 513, A57

Zsom, A., Ormel, C. W., Dullemond, C. P., \& Henning, T. 2011, A\&A, 534, A73 


\section{Appendix A: Geometric model for the computation of the effective stress}

The geometric approach to the deformation theory of hot pressing and the conclusions for the plastic flow have been investigated by Kakar \& Chaklader (1967) and Rao \& Chaklader (1972). However, the detailed derivation of the formulae was not provided. Thus, we present the theory in detail and improve some aspects that were treated in a simplified manner in the studies mentioned above.

In the study by Kakar \& Chaklader (1967) four basic systems of packing are considered, defined by the geometric arrangement of the grains and by the coordination number (this is the number $Z$ of the contact points, an average value for equally sized spheres). In the simple cubic packing (SCP) every grain has four neighbours in a horizontal layer and two additional neighbours in the layers above and below. Orthorhombic (ORP) packing corresponds to six neighbours in a layer (plus two above and below). The rhombohedral packing (RHP) has coordination number 12 and the body-centred cubic packing (BCCP) 8. Although in a unidirectional force field the body-centred cubic packing is an unstable arrangement, we consider this case for the sake of completeness. Furthermore, although stability varies with the packing type, we assume that the symmetry is sustained throughout the whole compaction process. Assuming homogeneous and isotropic composition of the grains and symmetrical spread upon application of stress, for different packings the spheres will deform to polyhedra upon complete compaction, e.g. cubes for SCP, hexagonal prisms for ORP, rhombic dodecahedra for RHP, and tetrakaidecahedra for BCCP. During the deformation, necks develop at the contact points. The form of the grains will change, whereas the volume will remain constant.

For each kind of packing a specific unit cell of a certain form which is occupied by a dust particle is considered. The form of a unit cell is defined by the polyhedra mentioned above. From Figs. A.2-A.4, the volume $V$ of such a unit cell can be computed. For SCP, this is the volume of a cube, and, since $y=\sqrt{r^{2}-a^{2}}$, we obtain

$V=(2 y)^{3}=8\left(r^{2}-a^{2}\right)^{\frac{3}{2}}$.

For ORP, the volume of a hexagonal prism is

$V=\frac{3 \sqrt{3}}{2}\left(\frac{4}{\sqrt{3}} y\right)^{2}(2 y)=4 \sqrt{3}\left(r^{2}-a^{2}\right)^{\frac{3}{2}}$.

For RHP we compute

$V=\frac{(2 y)^{3}}{\sqrt{2}}=4 \sqrt{2}\left(r^{2}-a^{2}\right)^{\frac{3}{2}}$,

and for BCCP

$V=\frac{8 \sqrt{2}}{27}\left(\sqrt{6} y_{1}\right)^{3}=\frac{32 \sqrt{3}}{9}\left(r^{2}-a_{1}^{2}\right)^{\frac{3}{2}}$.

Thus, the volume of the unit cell can be expressed as the product of a constant $\beta_{1}$ (the pre-factor of the bracket) and the power $\left(r^{2}-a^{2}\right)^{3 / 2}$.

The deformation of the BCC packing proceeds in two stages. For this reason we describe the theory for the other cases and the first stage of the BCCP deformation first, and then consider the second deformation stage of the BCC packing separately.

Prior to the hot pressing, the grain touches the surface of the unit cell at $Z$ points where the neighbouring grains touch

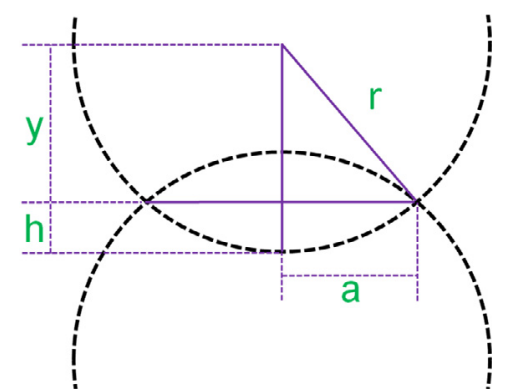

Fig. A.1. General deformation geometry at any contact point between two grains.

each other $\left(Z_{1}\right.$ for BCCP). During compaction, the grains deform plastically at the contact points and develop necks. The cross-section of such a neck between two grains has a circular form and is called the face (the contact points have evolved to contact cross-sections). Still, the volume of the particle needs to be kept constant. Thus, the current radius of the grain is assumed to increase and the volume of those parts of the grain now lying outside of the unit cell (the caps) need to be subtracted from the current sphere volume (we note that the initial sphere volume is normalised to 1 ):

$1=$ const. $=\frac{4}{3} \pi r_{0}^{3}=\frac{4}{3} \pi r^{3}-Z V_{\text {cap }}$,

where $r$ is the current grain radius and

$V_{\text {cap }}=\pi \frac{h^{2}}{3}(3 r-h)$

is the volume of a cap. Using the relation $h=r-y$ defined by the geometry of the packings (Fig. A.1), we obtain the normalised volume of the particle for the first three packings as

$$
\begin{aligned}
1 & =\frac{4}{3} \pi r_{0}^{3}=\frac{4}{3} \pi r^{3}-Z V_{\text {cap }} \\
& =\frac{4}{3} \pi r^{3}-\frac{Z \pi}{3}(r-y)^{2}(2 r+y) \\
& =\frac{4}{3} \pi r^{3}-\frac{Z \pi}{3}\left(2 r^{3}-2 r^{2} \sqrt{r^{2}-a^{2}}-a^{2} \sqrt{r^{2}-a^{2}}\right) \\
& =\frac{4}{3} \pi r^{3}-\frac{Z \pi}{3} 2 r^{3}+\frac{Z \pi}{3}\left(2 r^{2}+a^{2}\right) \sqrt{r^{2}-a^{2}} \\
& =\frac{Z \pi}{3}\left(2 r^{2}+a^{2}\right)\left(r^{2}-a^{2}\right)^{\frac{1}{2}}-\frac{(2 Z-4) \pi}{3} r^{3} \\
& =\alpha\left(2 r^{2}+a^{2}\right)\left(r^{2}-a^{2}\right)^{\frac{1}{2}}-\beta r^{3} \\
& =r^{3}\left(\alpha\left(2+\left(\frac{a}{r}\right)^{2}\right)\left(1-\left(\frac{a}{r}\right)^{2}\right)^{\frac{1}{2}}-\beta\right) .
\end{aligned}
$$

This defines the geometric constants $\alpha$ and $\beta$ from Table 1 as $\alpha=Z \pi / 3$ and $\beta=(2 Z-4) \pi / 3$. From Eq. (A.7) the current radius of the particle is computed:

$$
r=\left[\alpha\left(2+\left(\frac{a}{r}\right)^{2}\right)\left(1-\left(\frac{a}{r}\right)^{2}\right)^{\frac{1}{2}}-\beta\right]^{-\frac{1}{3}} .
$$

Given the ratio $a / r$, the current radius $r$ of the sphere during deformation can be found (in arbitrary units corresponding to 

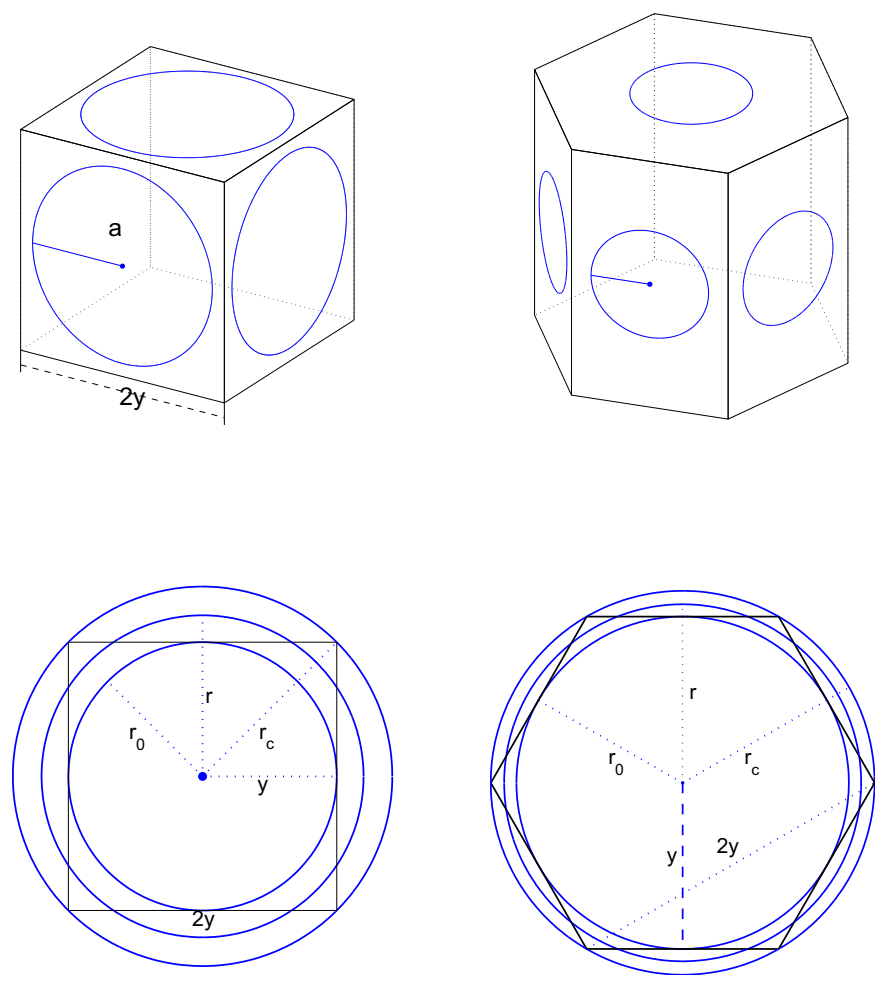

Fig. A.2. Unit cells and their intersections with an inscribed grain for the SCP and the ORP (top row from left to right) along with the top views of the unit cells and of the grain at its initial and critical (final) stages of deformation (bottom row).

unit volume of the sphere). The ratio $a / r$ is computed from the expression for the bulk density $D$, defined as the ratio of the volume of the particle to the volume of the unit cell. This last value is $\beta_{1}\left(r^{2}-a^{2}\right)^{3 / 2}$ (see Table 1$)$. From the normalised grain volume 1 and the volume of the unit cell we obtain

$$
D=\frac{1}{\beta_{1}\left(r^{2}-a^{2}\right)^{\frac{3}{2}}} \text {. }
$$

Prior to compaction, i.e. for $a=0$, the radius equals the initial radius $\left(r=r_{0}\right)$, hence

$$
D=D_{0}=\frac{1}{\beta_{1} r_{0}^{3}}
$$

holds, thus,

$$
\begin{aligned}
\frac{1-\phi_{0}}{1-\phi} & =\frac{D_{0}}{D}=\left[\frac{\left(r^{2}-a^{2}\right)}{r_{0}^{2}}\right]^{\frac{3}{2}}=\left[\left(\frac{r}{r_{0}}\right)^{2}-\left(\frac{a}{r_{0}}\right)^{2}\right]^{\frac{3}{2}} \\
& =\left(\frac{r}{r_{0}}\right)^{3}\left[1-\left(\frac{a}{r}\right)^{2}\right]^{\frac{3}{2}} .
\end{aligned}
$$

This is solved for $(a / r)^{2}$ :

$\left(\frac{a}{r}\right)^{2}=1-\left(\frac{r_{0}}{r}\right)^{2}\left(\frac{1-\phi_{0}}{1-\phi}\right)^{\frac{2}{3}}$.

For the computation of the effective stress, we also need the area of the cross-section of the unit cell. In terms of the geometries shown in Figs. A.2-A.4, the cross-section of a unit cell is the area of its projection onto the horizontal plane. For SCP this is a square with the edge length $2 y$, for ORP a regular hexagon with the edge length $(2 / \sqrt{3}) y$, for RHP a square with the edge length $2 y$, and for BCCP an irregular octagon with four edges of the length $\sqrt{2 / 3} y_{1}$, four edges of the length $(2 / \sqrt{3}) y_{1}$ and the internal angle of 135 degrees. The areas of those polygons can be written as $\gamma y^{2}$ (or as $\gamma y_{1}^{2}$ for BCCP; see Table 1 for the values of $\gamma$ ).

If a load $m$ acts on a face then the stress on the face is equal $\mathrm{m} /\left(\gamma y^{2}\right)$. The total stress on the sphere is $Z m /\left(\gamma y^{2}\right)$. In a planetesimal, the applied stress is the lithostatic pressure $\sigma_{0}$ and is equal to the stress on the face:

$\sigma_{0}=\frac{m}{\gamma y^{2}}$

From this the load acting on the cross-section of the unit cell follows as $m=\sigma_{0} \gamma y^{2}$ and can be divided into two components. It is equal to the sum of the load acting on the sphere through $N$ non-vertical flat faces $\pi a^{2} \cos (\theta) \sigma_{1}$ lying above the equator of the unit cell and of the load acting on the void space $c \sigma_{2}$,

$\gamma y^{2} \sigma_{0}=N \pi a^{2} \cos (\theta) \sigma_{1}+c \sigma_{2}$,

where $\pi a^{2}$ is the area of the neck, $\theta$ is the inclination angle of the faces, $c$ is the cross-section of the porous part of the unit cell, $\gamma$ is a geometric constant which depends on the packing (see Table 1 ), $\sigma_{1}$ is the stress acting along the $z$-axis through the neck, and $\sigma_{2}=0$ is the stress on the void space. The last equation is solved for $\sigma_{1}$ to obtain

$\sigma_{1}=\frac{\gamma}{N \pi \cos (\theta)}\left(\frac{y}{a}\right)^{2} \sigma_{0}=: \frac{1}{\alpha_{1}} \frac{r^{2}-a^{2}}{a^{2}} \sigma_{0}$.

Above we have shown $D=\beta_{1}^{-1}\left(r^{2}-a^{2}\right)^{-3 / 2}$. From this we compute

$$
\begin{aligned}
D & =\frac{1}{\beta_{1}\left(r^{2}-a^{2}\right)^{\frac{3}{2}}} \\
& \Leftrightarrow r^{2}-a^{2}=\frac{1}{D^{\frac{2}{3}} \beta_{1}^{\frac{2}{3}}} \\
& \Leftrightarrow \frac{1}{r^{2}-a^{2}}=\beta_{1}^{\frac{2}{3}} D^{\frac{2}{3}} \\
& \Leftrightarrow \frac{r^{2}}{r^{2}-a^{2}}=r^{2} \beta_{1}^{\frac{2}{3}} D^{\frac{2}{3}} \\
& \Leftrightarrow \frac{r^{2}-a^{2}+a^{2}}{r^{2}-a^{2}}=r^{2} \beta_{1}^{\frac{2}{3}} D^{\frac{2}{3}} \\
& \Leftrightarrow 1+\frac{a^{2}}{r^{2}-a^{2}}=r^{2} \beta_{1}^{\frac{2}{3}} D^{\frac{2}{3}} \\
& \Leftrightarrow \frac{a}{r^{2}-a^{2}}=D^{\frac{2}{3}} \beta_{1}^{\frac{2}{3}} r^{2}-1 .
\end{aligned}
$$

Thus,

$\sigma_{1}=\frac{1}{\alpha_{1}} \frac{1}{D^{\frac{2}{3}} \beta_{1}^{\frac{2}{3}} r^{2}-1} \sigma_{0}$.

To obtain the particle volume in the second deformation stage of the BCCP, one needs to subtract $Z_{1}=8$ caps of the volume 

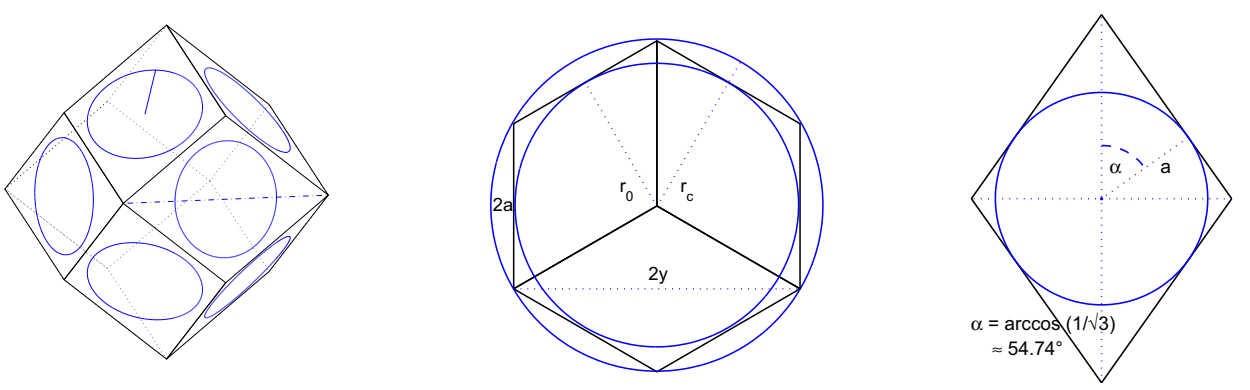

Fig. A.3. Unit cell of the RHP and its intersections with an inscribed grain (left panel), the top right view of the unit cell and of the grain at its initial and critical (final) stage of deformation (central panel), and a single face of the unit cell at the critical stage of the deformation (right panel).
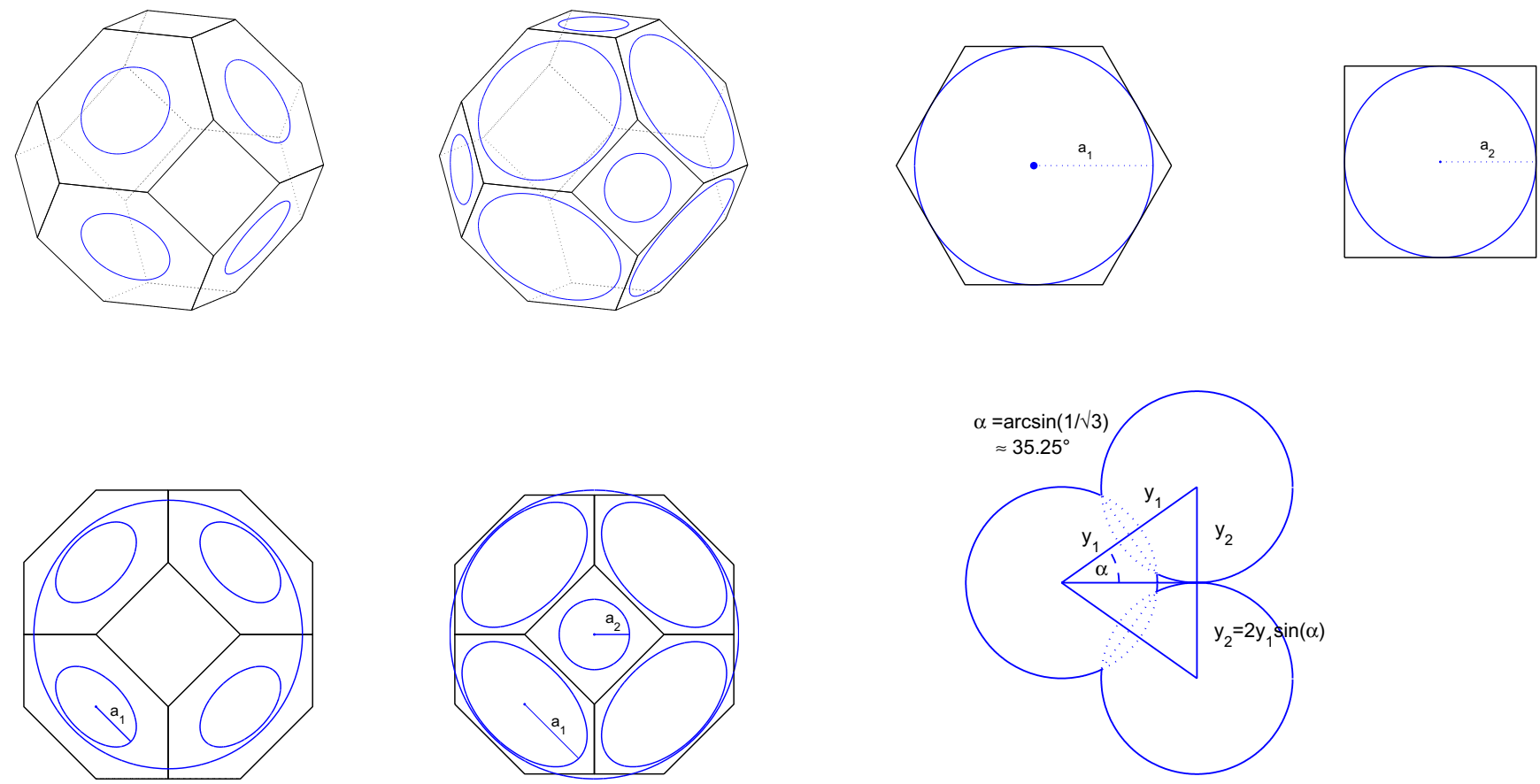

Fig. A.4. Unit cells and their intersections with an inscribed grain for the two deformation stages of the BCCP (top row, first and second panels); the faces of the unit cell at the second critical deformation stage (top row, third and fourth panels); topview of the unit cell before the first critical stage (bottom row, left panel) and after the first but before the second critical stage (bottom row, central panel); and the arrangement of the spheres at the first critical deformation stage as seen in the plane defined by the centres of the three spheres (bottom row, right panel).

$V_{\text {cap }, 1}=\pi \frac{h_{1}^{2}}{3}\left(3 r-h_{1}\right)$ and additionally $Z_{2}=6$ caps of the volume $V_{\text {cap }, 2}=\pi \frac{h_{2}^{2}}{3}\left(3 r-h_{2}\right)$ :

$$
\begin{aligned}
1 & =\frac{4}{3} \pi r_{0}^{3}=\frac{4}{3} \pi r^{3}-Z_{1} V_{\text {cap }, 1}-Z_{2} V_{\text {cap }, 2} \\
& =\frac{4}{3} \pi r^{3}-Z_{1} \pi \frac{h_{1}^{2}}{3}\left(3 r-h_{1}\right)-Z_{2} \pi \frac{h_{2}^{2}}{3}\left(3 r-h_{2}\right) \\
& =\sum_{i=1,2} \frac{Z_{i} \pi}{3}\left(2 r^{2}+a_{i}^{2}\right)\left(r^{2}-a_{i}^{2}\right)^{\frac{1}{2}}-\frac{\left(2 Z_{1}+2 Z_{2}-4\right) \pi}{3} r^{3} \\
& =\sum_{i=1,2} \alpha_{0, i}\left(2 r^{2}+a_{i}^{2}\right)\left(r^{2}-a_{i}^{2}\right)^{\frac{1}{2}}-\beta r^{3} \\
& =r^{3}\left(\sum_{i=1,2} \alpha_{0, i}\left(2+\left(\frac{a_{i}}{r}\right)^{2}\right)\left(1-\left(\frac{a_{i}}{r}\right)^{2}\right)^{\frac{1}{2}}-\beta\right)
\end{aligned}
$$

This defines the geometric constants $\alpha_{0,1}=\frac{Z_{1} \pi}{3}, \alpha_{0,2}=\frac{Z_{2} \pi}{3}$ and $\beta=\frac{\left(2 Z_{1}+2 Z_{2}-4\right) \pi}{3}$ for BCCP. Similarly to the other cases, Eq. (A.18) implies

$r=\left[\sum_{i=1,2} \alpha_{0, i}\left(2+\left(\frac{a_{i}}{r}\right)^{2}\right)\left(1-\left(\frac{a_{i}}{r}\right)^{2}\right)^{\frac{1}{2}}-\beta\right]^{-\frac{1}{3}}$.
The power $\left(a_{1} / r\right)^{2}$ is computed analogously to Eq. (A.12):

$$
\left(\frac{a_{1}}{r}\right)^{2}=1-\left(\frac{r_{0}}{r}\right)^{2}\left(\frac{1-\phi_{0}}{1-\phi}\right)^{\frac{2}{3}}
$$

For $\left(a_{2} / r\right)^{2}$ we consider the first critical stage $c_{1}$ when the cap of the second kind with the radius $a_{2}$ starts to form and express the relative density in the terms of $y_{2}$,

$D=\frac{1}{\frac{3 \sqrt{3}}{8} \beta_{1}\left(r^{2}-a_{2}^{2}\right)^{\frac{3}{2}}}$,

because $y_{1}=(\sqrt{3} / 2) y_{2}$. Then

$$
\begin{aligned}
\frac{1-\phi_{\mathrm{c}_{1}}}{1-\phi} & =\frac{D_{\mathrm{c}_{1}}}{D}=\frac{\frac{3 \sqrt{3}}{8} \beta_{1}\left(r^{2}-a_{2}^{2}\right)^{\frac{3}{2}}}{\frac{3 \sqrt{3}}{8} \beta_{1} r_{\mathrm{c}_{1}}^{3}}=\frac{\left(r^{2}-a_{2}^{2}\right)^{\frac{3}{2}}}{r_{\mathrm{c}_{1}}^{3}} \\
& =\left(\frac{r}{r_{\mathrm{c}_{1}}}\right)^{3}\left[1-\left(\frac{a_{2}}{r}\right)^{2}\right]^{\frac{3}{2}}
\end{aligned}
$$

and

$\left(\frac{a_{2}}{r}\right)^{2}=1-\left(\frac{r_{\mathrm{c}_{1}}}{r}\right)^{2}\left(\frac{1-\phi_{\mathrm{c}_{1}}}{1-\phi}\right)^{\frac{2}{3}}$ 
W. Neumann et al.: Modelling of compaction in planetesimals

Table A.1. Additional parameter values for the derivation of the effective stress.

\begin{tabular}{llllll}
\hline \hline & SCP & ORP & RHP & BCCP1 & BCCP2 \\
\hline$\alpha$ & $2 \pi$ & $\frac{8}{3} \pi$ & $4 \pi$ & $\frac{8}{3} \pi$ & $\alpha_{0,1}=\frac{8}{3} \pi$ \\
- & - & - & - & - & $\alpha_{0,2}=2 \pi$ \\
$\beta$ & $\frac{8}{3} \pi$ & $4 \pi$ & $\frac{20}{3} \pi$ & $4 \pi$ & $8 \pi$ \\
$\gamma$ & 4 & $2 \sqrt{3}$ & 4 & $\frac{14}{3}$ & $\frac{14}{3}$ \\
$\delta$ & $\frac{1}{\sqrt{2}}$ & $\frac{1}{2}$ & $\frac{1}{2}$ & $\frac{1}{2}$ & $\delta_{1}=\frac{1}{\sqrt{3}}$ \\
- & - & - & - & - & $\delta_{2}=\frac{1}{3}$ \\
$N$ & 1 & 1 & 4 & 4 & 5 \\
$\theta$ & 0 & 0 & $\arccos \left(\frac{\sqrt{2}}{\sqrt{3}}\right)$ & $\arccos \left(\frac{1}{\sqrt{3}}\right)$ & $\arccos \left(\frac{1}{\sqrt{3}}\right)$ \\
- & - & - & $\approx 35.26^{\circ}$ & $\approx 54.73^{\circ}$ & $\approx 54.73^{\circ}$ \\
\hline
\end{tabular}

Notes. See also Table 1.

follow. The relation between $a_{1}$ and $a_{2}$ is obtained from Eqs. (A.20) and (A.23) as

$a_{2}=\left[\frac{1-\left(\frac{r_{\mathrm{c}_{1}}}{r}\right)^{2}\left(\frac{1-\phi_{\mathrm{c}_{1}}}{1-\phi}\right)^{\frac{2}{3}}}{1-\left(\frac{r_{0}}{r}\right)^{2}\left(\frac{1-\phi_{0}}{1-\phi}\right)^{\frac{2}{3}}}\right]^{\frac{1}{2}} a_{1}=c(\phi) a_{1}$.

The load on the octagonal cross-section of the unit cell of the $\mathrm{BCCP}$ is

$\gamma y_{1}^{2}=4 \pi a_{1}^{2} \cos (\theta) \sigma_{1}+\pi a_{2}^{2} \sigma_{2}$,

where $\sigma_{1}$ is the effective stress on the faces of the first kind, $\sigma_{2}$ on the second kind, and $\cos (\theta)=1 / \sqrt{3}$. Assuming equal load per face, we obtain $\pi a_{1}^{2} \cos (\theta) \sigma_{1}=\pi a_{2}^{2} \sigma_{2}$ and from this

$\gamma y_{1}^{2} \sigma_{0}=\frac{N}{\cos (\theta)} \pi a_{1}^{2} \sigma_{1}$,

where $N=5$. Thus,

$\sigma_{1}=\frac{\gamma}{N \pi \cos (\theta)}\left(\frac{y_{1}}{a_{1}}\right)^{2} \sigma_{0}=\frac{1}{\alpha_{1}} \frac{r^{2}-a_{1}^{2}}{a_{1}^{2}} \sigma_{0}$.

The geometric relations described above are valid from the beginning of the deformation until the faces start to touch each other. In the beginning, for the normalised grain volume 1 the initial grain radius $r_{0}$ is equal to $(3 / 4 \pi)^{1 / 3}$. The stage when the equations lose their validity corresponds to a certain particle radius $r_{\mathrm{c}}$, the critical radius. This can be computed as follows. For the first four cases, the critical face radius $a$ can be expressed in terms of the critical particle radius as $a_{\mathrm{c}}=\delta r_{\mathrm{c}}$ with the constants $\delta$ (equal to $1 / \sqrt{2}, 1 / 2,1 / 2$, and $1 / 2$, respectively). Substituting this into Eq. (A.8) we obtain

$r_{\mathrm{c}}=\left[\alpha\left(2+\delta^{2}\right)\left(1-\delta^{2}\right)^{\frac{1}{2}}-\beta\right]^{-\frac{1}{3}}$.

From Eq. (A.10) we obtain the relative initial densities $D_{0}$ (see Table 1 ). The critical density $D_{\mathrm{c}}$ is obtained by substituting $r_{\mathrm{c}}$ and $a_{\mathrm{c}}=\delta r_{\mathrm{c}}$ into Eq. (A.9):

$D_{\mathrm{c}}=\beta_{1}^{-1}\left(r_{\mathrm{c}}^{2}-\delta^{2} r_{\mathrm{c}}^{2}\right)^{-\frac{3}{2}}=r_{\mathrm{c}}^{-3} \beta_{1}^{-1}\left(1-\delta^{2}\right)^{-\frac{3}{2}}$.

At the transition from the first to the second deformation stage of the BCC packing, contact points between the particle and the six rectangular faces of the unit cell form. Using $\delta_{1}=1 / 2$, the particle radius at this moment of deformation $r_{\mathrm{c}_{1}}$ and the first critical density $D_{\mathrm{c}_{1}}$ follow from Eqs. (A.8) and (A.9), or using $\delta_{1}=0.5$ and $a_{2}=0$, from Eqs. (A.19) and (A.21), respectively. At the end of the second stage, all fourteen faces come in contact. Here, with $\delta_{1}=1 / \sqrt{3}$ and $\delta_{2}=1 / 3$, the second critical radius $r_{\mathrm{c}_{2}}$ and the second critical density $D_{\mathrm{c}_{2}}$ follow from Eqs. (A.19) and (A.21).

After the respective critical density is reached, the geometric approach to the computation of the effective stress $\sigma_{1}$ can no longer be applied (i.e. for $D \geq D_{\mathrm{c}}$ or $\left.D \geq\left(D_{\mathrm{c}}\right)_{2}\right)$. In this case we use a continuous linear relationship between $\left(D_{\mathrm{c}}, \sigma_{1}\left(D_{\mathrm{c}}\right)\right)$ and $\left(1.0, \sigma_{0}\right)$. 